\title{
ARTICLE Non-toxigenic Bacteroides fragilis (NTBF) administration reduces bacteria-driven chronic colitis and tumor development independent of polysaccharide A
}

June L. Chan ${ }^{1,2}$, Shaoguang Wu ${ }^{2}$, Abby L. Geis ${ }^{3,8}$, Gabrielle V. Chan ${ }^{2}$, Talles A. M. Gomes ${ }^{4}$, Sarah E. Beck ${ }^{5}$, Xinqun Wu ${ }^{2}$, Hongni Fan ${ }^{3,6}$, Ada J. Tam ${ }^{3,6}$, Liam Chung ${ }^{3,6,7}$, Hua Ding ${ }^{2}$, Hao Wang ${ }^{2,6}$, Drew M. Pardoll ${ }^{3,6}$, Franck Housseau ${ }^{3,6}$ and Cynthia L. Sears

Polysaccharide A (PSA), an immunogenic capsular component of non-toxigenic Bacteroides fragilis (NTBF) strain NCTC 9343, is reported to promote mucosal immune development and suppress colitis. Contrastingly, enterotoxigenic Bacteroides fragilis (ETBF) is highly associated with inflammatory bowel disease (IBD) and colorectal cancer (CRC), rapidly inducing IL-17-dependent murine colitis and tumorigenesis. In specific-pathogen-free (SPF) C57BL/6 wild-type (WT) and multiple intestinal neoplasia (Min ${ }^{A p c 716+/-}$ ) mice, we show that sequential treatment of the NTBF strain, 9343, followed by the ETBF strain, 86-5443-2-2 (86), diminished colitis and tumorigenesis. Mice treated simultaneously with 9343 and 86 exhibited both severe colitis and tumorigenesis. Abrogated disease severity in sequentially treated mice was attributed to 9343 strain dominance and decreased IL-17A, but 86 colonization prior to or simultaneous with 9343 mitigated the anti-inflammatory effect of 9343. Remarkably, 9343-mediated protection was independent of PSA, as sequentially treated mice receiving $\triangle \mathrm{PSA} 9343$ exhibited similar protection. Further, SPF WT and Min mice colonized with PSA-competent or PSA-deficient 9343 exhibited similar IL-10, IL-17, and IFN- $\gamma$ responses. Treatment of 86-colonized mice with 9343 failed to disrupt 86 pathogenesis. Our findings demonstrate that 9343 colonization, independent of PSA, offers prophylaxis against colitis-inducing 86 but may not be a valid therapy once colitis is established.

Mucosal Immunology (2019) 12:164-177; https://doi.org/10.1038/s41385-018-0085-5

\section{INTRODUCTION}

In the United States, colorectal cancer (CRC) is the second leading cause of cancer-related deaths and is the fourth most common malignant neoplasm among both males and females. ${ }^{1}$ Yet worldwide, CRC cases are also emerging in locations previously considered to be low risk, including South America, Eastern Europe, and Eastern Asia. ${ }^{2}$ This rise in global incidence is likely, in part, due to globalization and its far-reaching impacts on dietary patterns, physical inactivity, obesity, and other risk factors. ${ }^{2,3}$ Alarmingly, CRC risk and mortality has also begun to rise in the young $(<50$ years old), now exhibiting double the risk for colon cancer and quadruple the risk of rectal cancer compared with the 1890 birth cohort. ${ }^{4,5}$ Cancer development is frequently induced or accelerated by chronic inflammation and, indeed, clinical inflammatory bowel disease (IBD) is another risk factor for CRC development. $^{6}$ Collectively, it is clear that augmented, population-accessible approaches to CRC prevention are needed.

Probiotics, defined as living microorganisms that confer a health benefit to the host when administered in adequate amounts, ${ }^{7}$ are proposed for disease prevention and therapy. Clinical studies assessing the effectiveness of probiotics as supplemental treatments in IBD and CRC have shown promise. For instance, single bacterial species, such as Escherichia coli Nissle 1917, or multispecies cocktails, such as VSL\#3, aid in the prevention or recurrence of post-operative pouchitis and in active ulcerative colitis therapy, ${ }^{8}$ a type of IBD. In CRC, oral treatment with Lactobacillus casei Shirota resolved atypia of colon tumors in patients who underwent colon resection. ${ }^{9}$ The human colon symbiote non-toxigenic Bacteroides fragilis (NTBF), strain NCTC 9343 , has been proposed as a probiotic. This is due to its reported anti-inflammatory actions in mice which include the initiation of regulatory T-cell expansion and production of interleukin (IL)-10, ${ }^{10}$ a characteristic immunosuppressive cytokine, and its hindering of pathogenic T helper 17 (Th17) cell development. ${ }^{11}$ These antiinflammatory actions have been ascribed to a capsular component, the polysaccharide A (PSA) molecule derived from the NTBF 9343 strain.

In contrast, enterotoxigenic Bacteroides fragilis (ETBF) strains secrete the $B$. fragilis toxin, $\mathrm{BFT}$, and induce marked colonic inflammation and tumor development in mice. ${ }^{12-14}$ BFT, a $20 \mathrm{kDa}$ zinc metalloprotease, severely alters cell-to-cell adherens junctions in the colon epithelium through the cleavage of E-cadherin,

\footnotetext{
${ }^{1}$ Department of Molecular Microbiology and Immunology, Bloomberg School of Public Health, Johns Hopkins University, Baltimore, MD, USA; ${ }^{2}$ Department of Medicine, Johns

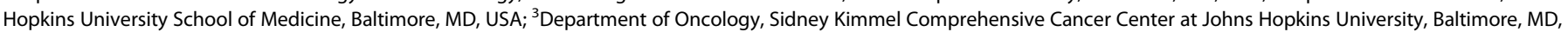

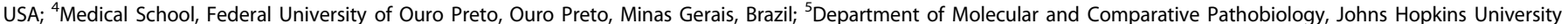

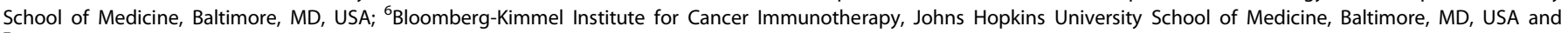
${ }^{7}$ Translational Tissue Engineering Center, Wilmer Eye Institute and Department of Biomedical Engineering, Johns Hopkins University, Baltimore, MD, USA Correspondence: Cynthia L. Sears (csears@jhmi.edu)

${ }^{8}$ Present address: Department of Microbiology and Immunology, Arkansas College of Osteopathic Medicine, Fort Smith, Arkansas, USA
}

Received: 5 April 2018 Revised: 1 August 2018 Accepted: 16 August 2018

Published online: 2 October 2018 
disrupting barrier function and stimulating the WNT signaling pathway to induce proto-oncogenes and cell proliferative genes, such as c-Myc. ${ }^{15,16}$ Moreover, a milieu of pro-inflammatory cytokines is produced, most notably the Th17 cytokine IL-17A, required for ETBF-induced tumorigenesis. ${ }^{14,17}$ Colonization with enterotoxigenic strains of $B$. fragilis is significantly higher in individuals with spontaneous $\mathrm{CRC}_{1}^{18}$ as well as in biofilms associated with tumors in individuals from familial adenomatous polyposis families. ${ }^{19}$ It is thus an enticing prospect that probiotic organisms may disrupt the cycle of ETBF pathogenic signaling, lessening the disease burden.

Humans are commonly colonized with $B$. fragilis, having a known proclivity for the colon mucosa. As the ETBF strain 86 and NTBF strain 9343 have been studied the most extensively, we tested the hypothesis that NTBF strain 9343 can protect against ETBF strain 86-mediated disease as an immunoregulatory probiotic. We found that when 9343 colonized the colon at high levels, prior to 86 treatment, the 9343 strain limited 86-induced inflammation and tumor development in mice. However, this protective effect was independent of 9343-encoded PSA or direct host immune system modulation and, rather, appeared solely mediated by competitive exclusion and mucosal 9343 strain dominance. Therapeutic 9343 treatment, of mice stably colonized with 86 , failed to diminish colitis or 86 dominance. Together, our results question the capacity of 9343-derived PSA to inhibit gut immune dysregulation and suggest that the action of NTBF strain 9343 as an anti-inflammatory probiotic is restricted to the prophylactic setting, acting only antecedent to ETBF strain 86 colonization.

\section{RESULTS}

The 9343 strain subverts 86 -induced inflammation and adenoma development independently of PSA

Colonization of specific-pathogen-free (SPF) C57BL/6 wild-type $\left(\mathrm{WT}, \mathrm{ApC}^{+/+}\right)$mice with ETBF, strain 86 , induces an acute, selflimiting inflammatory diarrhea and robust mucosal IL-17A response. ${ }^{12,13}$ After 3 to 5 days, the mice recover from the diarrhea but maintain subclinical, chronic Th17-mediated colitis for about 1 year after infection. ${ }^{13,20}$ As 9343 -derived PSA has been shown to be protective in the 2,4,6-trinitrobenzenesulfonic acid (TNBS) and Th17-inducing Helicobacter hepaticus SPF experimental colitis models, ${ }^{10,21}$ we sought to disrupt 86 -induced colitis by sequentially introducing 9343 followed by 86 (9343 $\rightarrow$ 86: 9343 then 863 days later) or by simultaneously introducing both strains $(9343+86: 9343$ and 86 at the same time) into SPF WT mice (S1 Fig, colitis protocol). The same co-infection treatments were replicated with the isogenic PSA mutant strain ( $\triangle \mathrm{PSA} 9343$ ) (S2 Fig). At 2 weeks post infection (p.i.), mice from both sequential treatment groups, $9343 \rightarrow 86$ and $\triangle P S A 9343 \rightarrow 86$, exhibited a significant decrease in $1 / 17 a$ transcription compared with $9343+86$ or 86-only mice as measured by whole colon quantitative reverse transcription-polymerase chain reaction (qRT-PCR; Fig. 1a). In contrast, $9343+86$-treated mice showed high $1 / 17 a$ gene expression, consistent with $86-$ only mice. However, both $9343 \rightarrow 86-$ and $\triangle$ PSA 9343 $\rightarrow$ 86-treated mice exhibited increased $1 / 17 a$ messenger RNA (mRNA) expression levels compared to 9343 or $\triangle$ PSA 9343 single colonization, indicating that inhibition of 86-induced Th17 responses was incomplete. Consistent with decreased inflammatory $1 / 17 a$ expression, $9343 \rightarrow 86$ and to a larger extent $\triangle$ PSA $9343 \rightarrow 86$ mice showed a decrease in histopathological inflammation and hyperplasia in comparison with 86-only mice (Fig. 1b, c). These findings suggest that 9343, irrespective of PSA expression, may offer at least partial protection against IL-17Adriven ETBF 86 colitis.

A prior report indicated that gnotobiotic (GNO) WT mice (originally germ free then colonized with 'known life') monocolonized with $\triangle$ PSA 9343 induces a pathogenic Th17 cell accumulation, a response suppressed by exogenous treatment with purified PSA. ${ }^{11}$ Notably, in our SPF WT mice, there were no differences in whole tissue $1117 a$ mRNA expression between 9343 and $\triangle \mathrm{PSA} 9343$ groups, either singly or sequentially treated, demonstrating that the absence of PSA did not drive a mucosal microenvironment high in IL-17A (Fig. 1a). NTBF 9343 colonization and PSA treatment have also been reported to induce $\mathrm{CD}^{+} \mathrm{T}$ cells to produce interferon (IFN)- $\gamma$ (GNO mice) $)^{22}$ and IL-10 (GNO and SPF mice $)^{10,21}$ indicative of its putative role in contributing to the Th1/Th2 balance and restriction of inflammatory responses in the gut. We compared the expression of cytokines typical of regulatory $\mathrm{T}$ (Treg) or $\mathrm{Tr} 1$ (IL-10) and Th1 (IFN- $\gamma$ ) responses in our SPF WT infection model. There were no distinct increases in transcriptional expression of the genes coding for IL-10 or IFN- $\gamma$ in SPF WT mice singly colonized with either 9343 or $\triangle$ PSA 9343 or co-infected with 86; modest increases were observed in singly colonized $\triangle P S A 9343$ mice that were significant compared to singly colonized 9343 mice, although these results remained close to baseline representing less than a twofold increase (Fig. 1a). Long-term assessment of SPF WT mice colonized with 9343 yielded no changes in characteristic Th cell cytokines (S3 Fig). These data suggest that in hosts with complex microbiota (e.g., SPF), the IFN- $\gamma$ and IL-10 cytokine response is not impacted by PSA introduction through 9343 colonization. Protection from 86 colitis is not mediated through 9343-derived PSA.

Since 9343 protected against 86 colitis, we next evaluated the role of 9343 during 86-induced tumorigenesis (S1 Fig, microadenoma/ macroadenoma protocol). The 86 infection of SPF Min $\left(\mathrm{ApC}^{+-}\right)$mice induces rapid colon tumorigenesis with microadenoma (detected by microscopy) onset at 5 days and quantifiable as early as 2 weeks, and macroadenomas visible as early as 4 weeks after colonization. ${ }^{14}$ Consistent with the results of the colitis model, SPF Min mice inoculated sequentially $(9343 \rightarrow 86$ or $\triangle P S A 9343 \rightarrow 86)$ showed a significant decrease in macroadenoma formation in comparison with $9343+86$ or 86 -only mice at 12 weeks (Fig. 2a, b). No differences in macroadenoma number were noted between 9343 and $\triangle \mathrm{PSA} 9343$ treatment groups, either singly or sequentially treated. Both 9343 and $\triangle$ PSA 9343 appear to prevent 86-induced tumor progression. Neither 9343 nor $\triangle$ PSA 9343 single colonization of Min mice increased macroadenoma formation in Min mice not infected with 86 and were comparable to Sham controls (Fig. 2a, b). The absence of increased macroadenomas in Min mice colonized with $\triangle P S A 9343$ again suggests that mucosal IL-17 is not increased in the absence of PSA, given that baseline tumorigenesis in Min mice is IL-17 dependent. ${ }^{23}$ When evaluating microadenomas at 2 to 4 weeks, we found that $9343 \rightarrow 86$ mice exhibited no significant reduction in microadenoma counts and microadenoma morphology and size remained variable, consistent with 86-only mice (Fig. 2c, d). Yet, it was observed that reduction of visible macroadenomas was maintained at 8, 12, and 16 weeks p.i. (S4 Fig). Although these results suggest that tumor initiation between co-inoculated groups (sequential or simultaneous) is similar, the 9343-mediated protection from tumor progression appears highly durable and suggests protection may be at the level of tumor growth versus tumor initiation. Both 9343 and $\triangle$ PSA 9343 appear to similarly prevent 86induced tumor progression. Distinct outliers were observed in sequentially treated $(9343 \rightarrow 86)$ Min and WT mice, e.g., mouse \#312 and mouse \#238 produced 51 and 10 macroadenomas, respectively (Fig. 2b). Given the mouse to mouse variability that can occur in cocolonization experiments, this finding prompted us to further evaluate the localization and relative burden of each $B$. fragilis strain in individual mice as a potential determinant of the effect of 9343 on 86-mediated colitis and tumorigenesis.

The 9343 strain-derived PSA does not impact $B$. fragilis colonization

In GNO mice, $B$. fragilis was reported to reside in the colonic crypts of the mucosa, ${ }^{24}$ with PSA as the purported mediator of this 

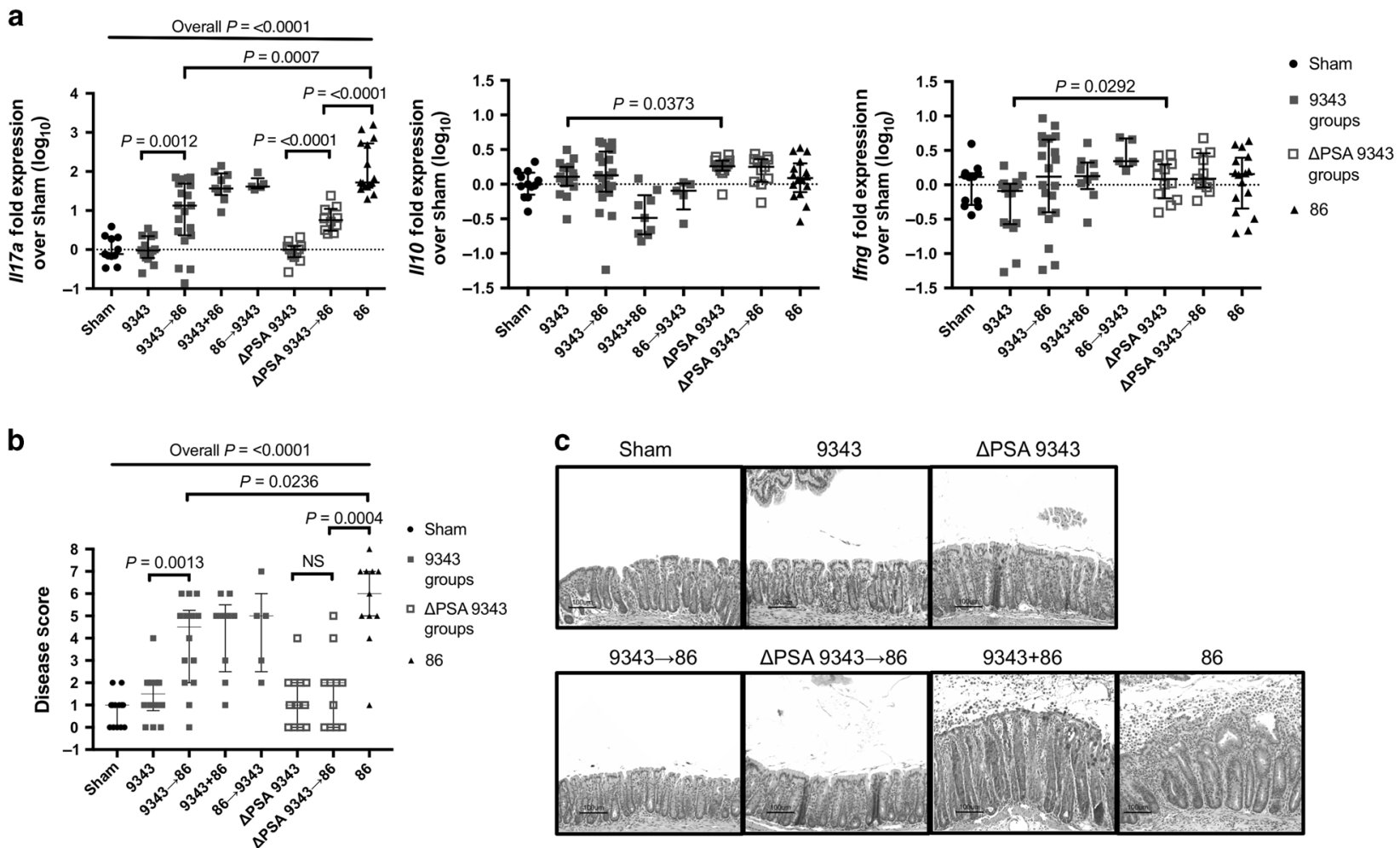

Fig. 1 PSA-competent and PSA-deficient 9343 incompletely reduce inflammatory responses. a-c SPF WT mice were inoculated sequentially $(9343 \rightarrow 86)(86 \rightarrow 9343)$ or simultaneously $(9343+86)$ and killed 2 weeks later. Distal colon tissue was harvested for whole tissue qRT-PCR. The remaining colon was reserved for FFPE. Bars indicate median and interquartile range. Data represent a compilation of 3 replicate experiments with 5 to 19 mice in total per group. a The $9343 \rightarrow 86$ and $\triangle$ PSA $9343 \rightarrow 86$ mice do not differ (NS) but exhibit reduced $/ / 17 a$ transcription compared with $9343+86(P=0.0284$ and $P=0.0003$, respectively) and 86 -only mice (see Figure) and elevated $I 117 a$ transcription compared to 9343 or $\triangle$ PSA 9343-alone mice (see Figure). In contrast, $9343+86$ vs 86 mice and 9343 vs $\triangle$ PSA 9343 mice do not differ (NS for both comparisons). $1 / 10$ and Ifng expression was similar between 9343 and $\triangle$ PSA 9343 single and co-inoculated mice but was increased in $\triangle$ PSA 9343 single colonized mice compared to 9343 mice, yet remaining close to baseline ( $<2$-fold increase). b Disease scores (additive hyperplasia and inflammation) of FFPE H\&E sections. The $9343 \rightarrow 86$ and $\triangle$ PSA $9343 \rightarrow 86$ mice exhibit lower disease scores compared with 86 control mice. c Representative H\&E histology depicting pronounced colon hyperplasia and inflammation in $9343+86$ and 86 control mice, whereas $9343 \rightarrow 86$ and $\triangle$ PSA $9343 \rightarrow 86$ mice are comparable to Sham or 9343 single colonized mice. Multiple group comparisons were conducted using Kruskal-Wallis testing and pairwise comparisons using Mann-Whitney $U$ testing

colonization through the suppression of antibacterial Th17 responses. ${ }^{11}$ Thus, in GNO mice, $\triangle$ PSA 9343 exhibited diminished mucosal colonization in comparison with the PSA-competent parental strain (9343). This colonization deficiency was restored through treatment with exogenous PSA or IL-17A neutralizing antibodies. ${ }^{11}$ We used fluorescence in situ hybridization (FISH) to localize $B$. fragilis in the mucosa of SPF and GNO mice, single colonized and monocolonized, respectively. In our studies, both 9343 and $\triangle$ PSA 9343 strains were observed to colonize in the lumen and at the mucosal surface in SPF and GNO mice, with a small subset visualized to reside in the mucus layer (Fig. 3a, arrows) (S5A Fig). B. fragilis penetration into the mucus layer appeared patchy and limited; a few instances of possible crypt invasion were also observed. In either housing condition, both bacterial strains exhibited the same colonization pattern. This finding is consistent with reports that $B$. fragilis can bind to, degrade, and utilize intestinal mucins as a nutrient source. ${ }^{25-27}$

$\triangle$ PSA 9343 monocolonized GNO mice were reported to display a significant decrease in the abundance of tissue-associated bacteria, suggestive that PSA is required for the close association of $B$. fragilis with the host mucosa. ${ }^{11}$ To test this assertion, the distal colon tissue and feces of SPF and GNO mice colonized with either 9343 or $\triangle P S A 9343$ were homogenized and cultured to quantify viable colony-forming units (CFUs) of bacteria closely associated with the epithelium (mucosal colonization) and in the stool (see Methods). No differences in the viable bacterial burden were observed in the feces or the mucosa at early or late time points ( 2 or 8 week) post infection (Fig. 3b). PSA deficiency did not change bacterial burden and localization, indicating that PSA does not routinely impact the parameters of $B$. fragilis niche occupation. Routine assessment of $B$. fragilis colonization in SPF mice also yielded similar fecal and mucosal colonization levels among 9343-, $\triangle P S A$ 9343-, and 86-treated mice (S6 Fig), with each strain achieving a similar colonization burden.

Disease outcome is determined by a critical threshold of 86 strain colonization

Strain 86 monocolonization induces rapid lethality, within 3 days, in GNO WT mice (129S6/SvEv ${ }^{28}$ and C57BL/6 (P. Fathi and C. Sears, unpublished data)). GNO mice (129S6/SvEv) colonized with 9343 and subsequently exposed to 86 survived $\sim 2$ weeks, but ultimately succumbed (S. Wu et al. unpublished data). Similarly, we showed that 86 IL-17A-mediated colitis and tumorigenesis were significantly abrogated in SPF sequentially infected mice $(9343 \rightarrow 86)$ (Figs. 1 and 2). Thus, we investigated whether B. fragilis strain competitive exclusion could be playing a role in disease prevention. In viable bacterial colony screenings of stools harvested from WT $9343 \rightarrow 86$ or WT $9343+86$-colonized mice, we found that one dominant strain was established as early as 1 week after infection. WT $9343 \rightarrow 86$ mice became colonized predominantly with 9343 (92\% of mice), while WT $9343+86$ mice became colonized predominantly with 86 (100\% of mice) (Fig. $4 a)$. 
a

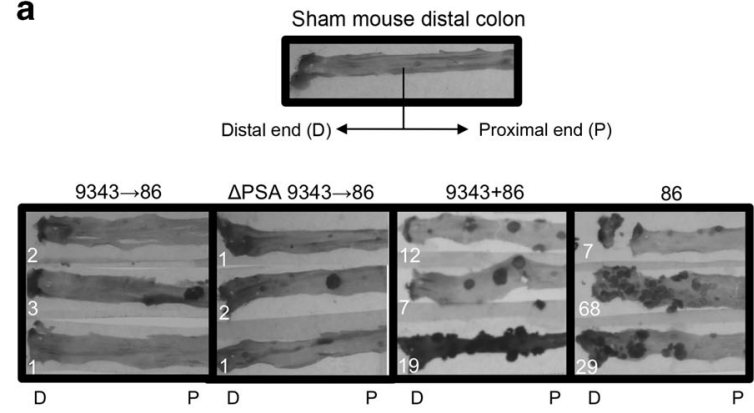

C

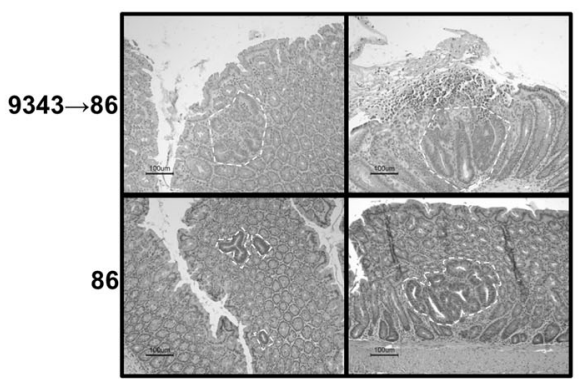

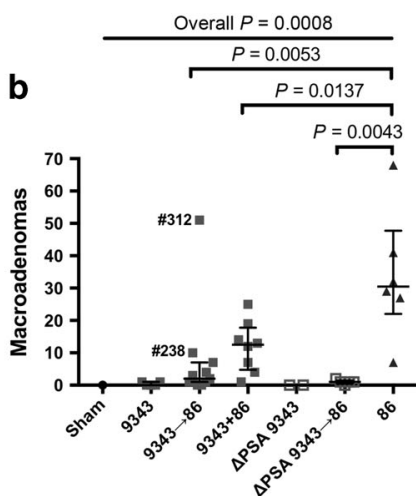

- Sham
- 9343
group$$
\triangle \mathrm{PSA} 9343
$$$$
\text { groups }
$$

- 86 Outlier $9343 \rightarrow 86$ Mouse distal colon

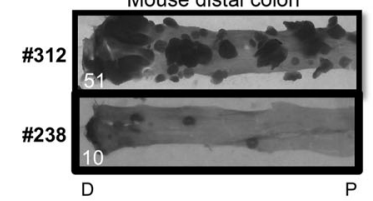

d

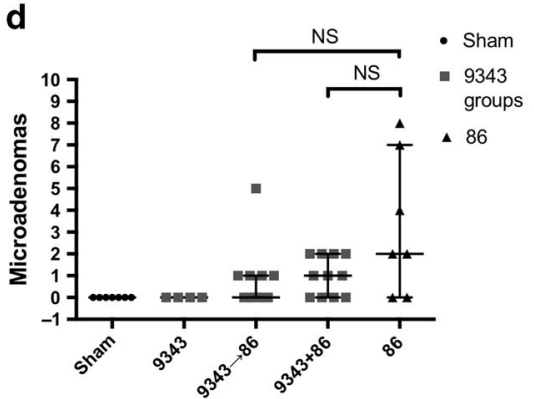

Fig. 2 PSA-competent and PSA-deficient 9343 similarly reduce adenoma development. a-d SPF Min mice were inoculated sequentially $(9343 \rightarrow 86)$ or simultaneously $(9343+86)$. Mice were killed at 12 weeks (macroadenomas) or 2 to 4 weeks (microadenomas). Bars indicate median and interquartile range. Macroadenomas: data shown are from 2 replicate experiments with 1 (Sham control only) to 11 mice in total per group. Microadenomas: data shown are from 1 experiment with 4 to 11 mice in total per group. a Images of representative methylene blue-stained distal colons. The $9343 \rightarrow 86$ and $\triangle P S A 9343 \rightarrow 86$ mice display decreased macroadenoma development compared with $9343+86$ and 86 control mice. b Entire colon macroadenoma quantification from individual mice. Both $9343 \rightarrow 86$ and $\triangle$ PSA $9343 \rightarrow 86$ mice exhibit a similar reduction in macroadenomas in comparison with $9343+86$ and 86 mice. Macroadenoma burden was similar between 9343 and $\triangle$ PSA 9343 mice. Image shows stained colons of $9343 \rightarrow 86$ high tumor producers: mouse \#312 and mouse \#238. c H\&E colon sections displaying variable microadenoma histopathology of $9343 \rightarrow 86$ and 86 control mice. d Quantification of microadenomas from individual mice. The $9343 \rightarrow 86$ mice do not display a significantly lower microadenoma burden compared with 86 control mice. Multiple group comparisons were conducted using Kruskal-Wallis testing and pairwise comparisons using Mann-Whitney $U$ testing

Using quantitative PCR (qPCR) to detect low-level colonization, the relative proportions of both $B$. fragilis strains were determined in the feces of co-inoculated mice. At 2 weeks p.i., WT $9343 \rightarrow 86$ and WT $\triangle$ PSA $9343 \rightarrow 86$ mice both showed dominance with 9343 strains in the feces (defined as $\geq 10^{5}$ detected copies) (purple and orange circles, Fig. 4b), whereas WT 9343+86 mice showed 86 dominance in the feces (green circles, Fig. 4b). Similarly, at $\geq 3$ months p.i., Min $9343 \rightarrow 86$ and Min $\triangle P S A$ 9343 $\rightarrow 86$ mice exhibited dominance with 9343 strains in the feces (purple and orange circles, Fig. 4c), whereas Min 9343+86 mice exhibited 86 dominance in the feces (green circles, Fig. 4c). Of note, we also observed that the high tumor producing Min 9343 $\rightarrow 86$ mice (\#312 and \#238 in Fig. 2b) showed complete 9343 displacement, indicative of the capability for host strain replacement by 86 with modification of disease trajectory. Strain quantification in distal colon mucosal tissue by qPCR mimicked the fecal results, although it approached the limits of detection (not shown).

The culture-based (Fig. 4a) and quantitative PCR (Fig. 4b, c) measures of strain dominance suggest two points: (1) 9343, given adequate colonization time, prevents 86 from establishing dominance, and (2) 86 exhibits a competitive advantage over 9343 during simultaneous co-infection. The percentage of WT and Min mice sequentially infected with $9343(9343 \rightarrow 86)$ and displaying a high 9343 copy number (i.e., high/low+high/high +9343 only) was $92.9 \%$ and $86.7 \%$, whereas those with a high 86 copy number (low/high + high/high +86 only) was $28.6 \%$ and $20.0 \%$, respectively (S1 and S2 Tables). These percentages exceed $100 \%$ because a subset of mice acquired high level colonization ( $\geq 10^{5}$ copies) with both 9343 and 86 . The percentage of WT and
Min mice simultaneously infected $(9343+86)$ and displaying a high 9343 copy number was $44.4 \%$ and $36.4 \%$, whereas those displaying a high 86 copy number was $100 \%$ and $90.9 \%$, respectively (S1 and S2 Tables). These data suggest that once a $B$. fragilis strain is established in the host, it is infrequently altered or supplanted by the introduction of another strain. Thus, in our SPF model, we show that a secondary $B$. fragilis strain introduced through subsequent challenge has the ability to maintain persistent colonization (up to 4 months), but at appreciably lower levels, on average, a 1000-fold less than the initial colonizing strain (Fig. 4b, c) (S1 and S2 Tables). The 86 strain dominance was also predictive of inflammatory and tumor responses. Strong positive correlations were observed between 86 copy number and $1117 a$ mRNA expression ( $r s=0.7469$ ) and tumor development ( $r s=$ 0.7899) (Fig. 4d, e). Of significance, in individual co-inoculated mice $(9343+86$ or $86 \rightarrow 9343)$ when the critical disease-triggering 86 burden $\left(\sim 10^{7}\right.$ copies) was achieved, relatively high levels of 9343 co-colonization ( $10^{5}-10^{7}$ copies) did not abrogate disease $\left({ }^{*}\right.$ denotes individual mice with $\geq 10^{5}$ copies of both 86 and 9343, Fig. 4b-e). Additionally, we observed the infrequent occurrence (2 out of 15 mice) of complete 9343 displacement by 86 in $9343 \rightarrow 86$ Min mice (purple circles: mouse \#312 \& \#238, Fig. 4c, e) that had been colonized long term. These data points correspond to the high tumor producers noted in Fig. $2 b$.

Collectively, these findings suggest that 9343 has the ability to prevent, yet not rescue, pathogenic 86 from reaching diseaseinducing levels within the host. Given the relatively high frequency of 9343 and 86 co-colonization observed, we postulated that 9343 and 86 might display distinct mucosal colonization 


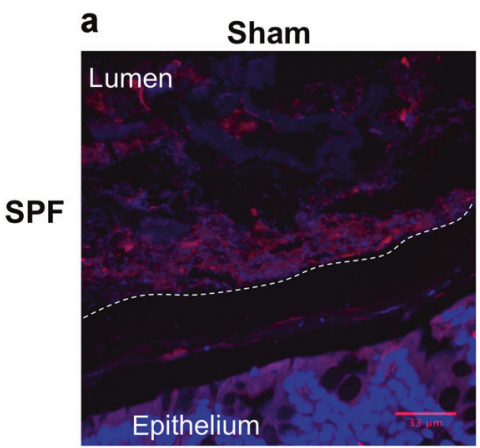

GF

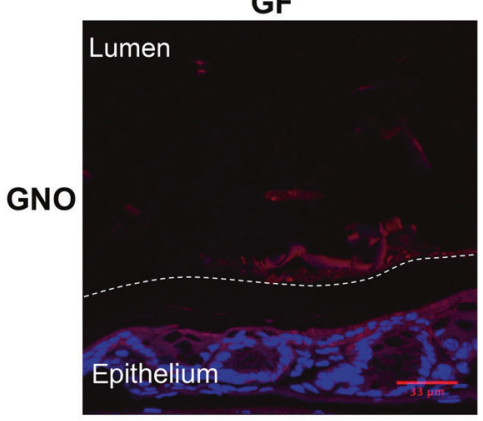

DAPI/9343(Сy3)

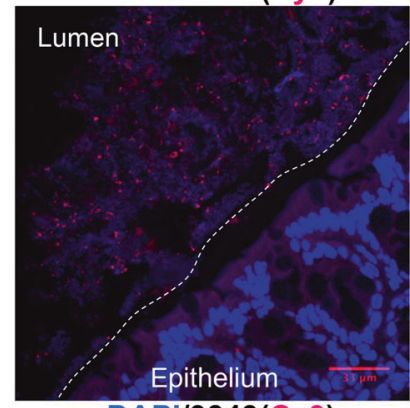

DAPI/9343(Cy3)

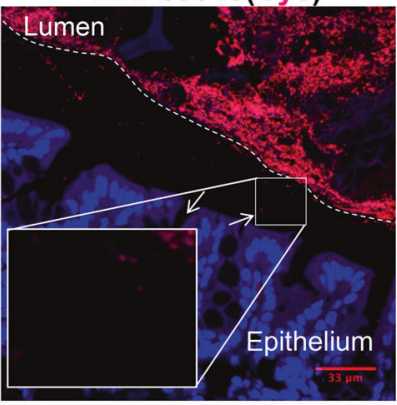

DAPI/APSA 9343(Су3)

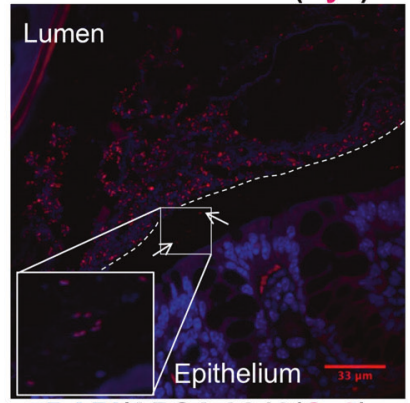

DAPI/DPSA 9343(Cy3)

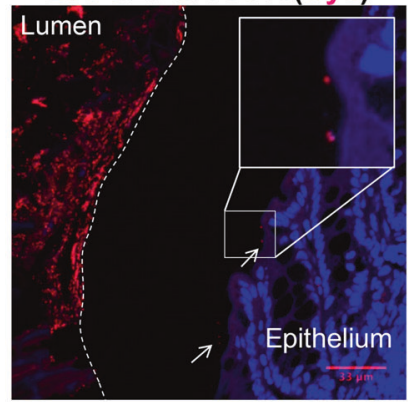

b

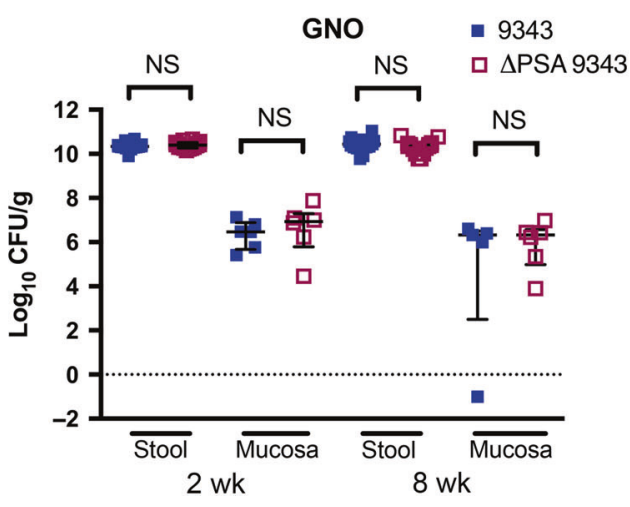

Fig. 3 PSA does not impact $B$. fragilis niche occupation. a, b SPF WT and GNO WT mice were colonized with 9343 or $\triangle$ PSA 9343 for 2 or 8 weeks. Mouse colons were unflushed, formalin-fixed, and FISH-stained: DAPI = blue, B. fragilis $16 \mathrm{~S}$ probe $=$ red. a Representative FISH-stained mouse colons ( 2 weeks p.i.); total magnification $\times 400$. Insets show images at total magnification $\times 630$. The stippled line indicates the border between the luminal contents and the epithelial mucus layer. The 9343 and $\triangle$ PSA 9343 strains colonize both the lumen and the mucosal surface with patchy mucus penetration (examples are shown with arrows). b Stool and mucosal colonization of mice inoculated with 9343 or $\triangle$ PSA 9343. Bars indicate median and interquartile range. Compiled data showing SPF WT stool: 2 to 18 in total per group; SPF WT mucosa: 2 to 10 in total per group; GNO WT stool: 15 to 16 in total per group; GNO WT mucosa: 5 to 6 in total per group

patterns. We sought to identify the colonic locale of both 9343 and 86 , using immunofluorescence (S7 Fig). Our results suggest both 9343 and 86 colonize the lumen and at the mucosal surface, with patchy mucus layer penetration (Fig. 4f) (S5B Fig). Mice cocolonized with 9343 and 86 also displayed localization to the lumen and at the mucosal surface, with no distinct preferences observed for either strain (Fig. 4f); patchy mucus layer penetration was also observed.

The 86 strain secretes an intraspecies inhibitory molecule Our data show that 86 consistently outcompetes 9343 during simultaneous co-infection in mice (Fig. $4 a-c$ ). Many factors, such as growth rate, nutrient utilization, colonization factors, and antimicrobial secretion, are vital mechanisms to microbial establishment and persistence. ${ }^{29}$ These elements may contribute to overall 86 fitness. To evaluate strain differences in growth and nutrient utilization, bacterial growth and recovery was monitored. Individually, 9343 and 86 strains exhibited similar growth rates, although the absence of PSA appeared to impact in vitro growth
(Fig. 5a). Intriguingly, when strains were co-cultured at equal starting points, the percent recovery of 9343 and $\triangle$ PSA 9343 was severely diminished after 24 to $48 \mathrm{~h}$ (Fig. 5b). Finally, when strains were co-cultured at varying input ratios for either 24 or 48 h, 9343 could only be recovered when it comprised the majority of the input strain (Fig. 5c). Since we did not observe growth differences between WT strains, we hypothesized that 86 was secreting an antimicrobial compound leading to 9343 growth inhibition.

Bacteriocins are small proteins that have a narrow killing spectrum, acting on closely related species or strains. ${ }^{30} \mathrm{We}$ conducted agar diffusion assays with cell-free 86 supernatants isolated from stationary phase cultures and found that, indeed, 86derived culture supernatants could inhibit the growth of 9343 and other closely related strains, independent of BFT. Protease treatment with trypsin, proteinase- $\mathrm{K}$, and heat inactivation at $100{ }^{\circ} \mathrm{C}$ eliminated 86 supernatant activity, indicating the inhibitory molecule is likely a protein (Fig. 5d) (S8 Fig). Proteins with membrane attack complex/perforin (MACPF) domains have been shown to mediate intraspecies killing in the gut Bacteroides. ${ }^{31,32}$ Of 
a

WT stool (1 wk)

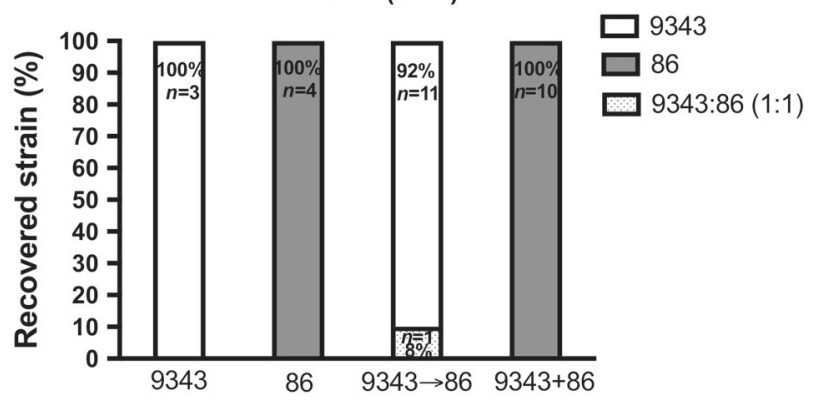

b

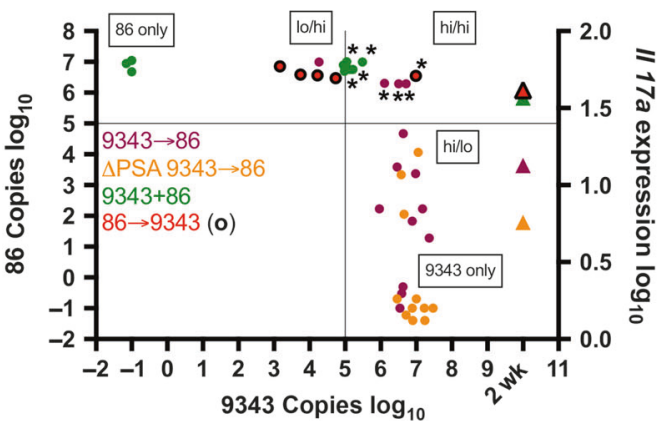

d

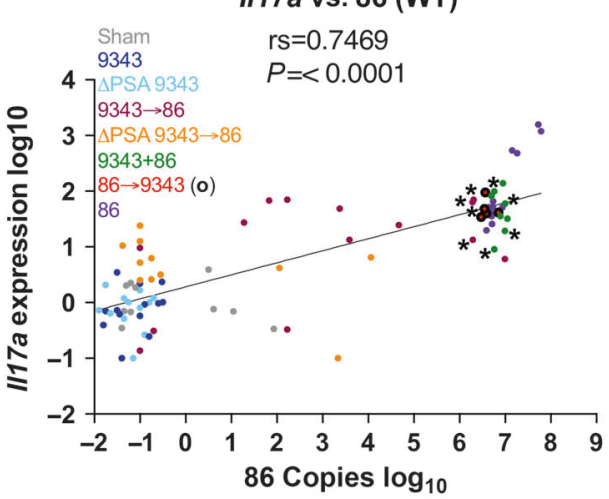

$\mathbf{f}$

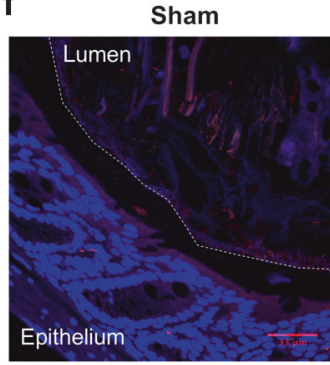

DAPI/9343(GFP)

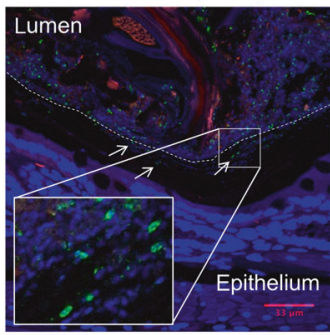

C

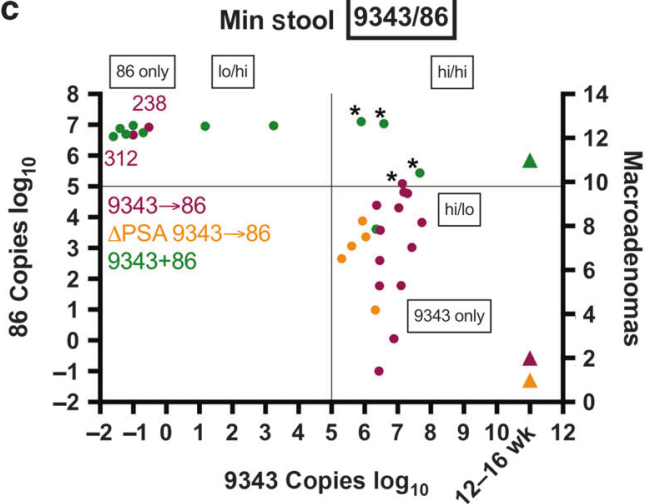

e

Macroadenoma vs. 86 (Min)
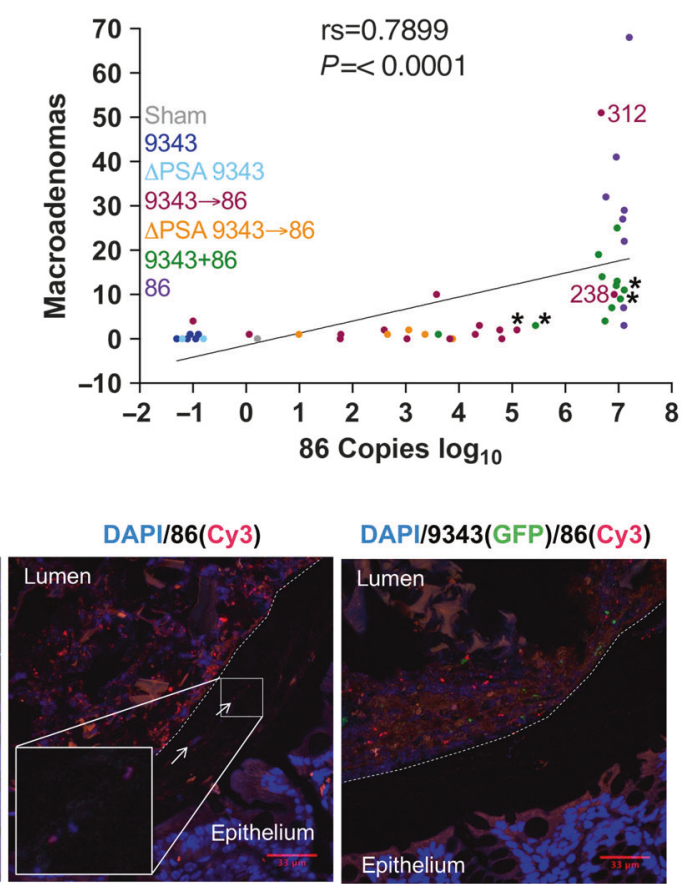

interest, 86 was found to carry an identical copy of the previously identified Bacteroidales-secreted antimicrobial protein 1 (BSAP-1) encoding gene, BF638R_1646, whereas 9343 lacks this gene. As expected, qRT-PCR analysis revealed that BF638R_1646 was expressed in 86 but not in 9343 (S9A Fig). As 9343 was previously shown to have BSAP-1 sensitivity, ${ }^{32}$ the secretion of this poreforming molecule is one likely candidate contributing to the 86 competitive advantage over 9343 , leading to dramatic growth inhibition of 9343.
Recently, a novel Bacteroides polysaccharide utilization locus regulating species saturation was described. ${ }^{24}$ Mutations in this commensal colonization factor locus $(c c f)$ altered $B$. fragilis niche occupation in mice, becoming permissive to challenge colonization by the same species. We hypothesized that differential gene expression in $c \subset f A$ and $C C f B$, the sigma/anti-sigma factor pair, would contribute to an 86 competitive advantage. However, distal colon ccf expression levels were similar between 9343 and 86 singly colonized SPF WT mice, suggesting that ccf gene 
Fig. 4 Bacterial strain dominance determines inflammatory and tumor responses. a Early evaluation of strain dominance. Viable CFU percentages of 9343 or 86 detected in stools harvested 1 week p.i. in SPF WT mice. Isolated B. fragilis colonies (up to 30) cultured from stool homogenates were randomly selected and screened by strain-specific PCR. In 11 of $129343 \rightarrow 86$ mice, only 9343 was detected (92\%); in 1 of 12 , both 9343 and 86 were detected in equal parts (8\%). In 10 of $109343+86$ mice, only 86 was detected (100\%). b-e Relative strain quantification (B. fragilis copies/100 ng of DNA) by fecal qPCR at 2 weeks (SPF WT) or 12-16 weeks (SPF Min) and presented in the order of 9343(NT)/86(ET): lo/hi, hi/hi, or hi/lo. Low (lo $=10^{1}<10^{5}$ copies. High (hi) $=\geq 10^{5}$ copies. Circles represent individual mice; triangles represent median $I / 17 a$ expression or tumor counts (see right vertical axis). ${ }^{*}$ Mice with hi/hi co-colonization levels. b Strain quantification in WT coinfected mice. c Strain quantification in Min co-infected mice. d Correlation of $1117 a$ expression with 86 copies in WT mice. e Correlation of tumor number with 86 copies in Min mice. f Representative IF staining in SPF mice ( 2 to 4 weeks p.i.); images taken at $\times 400$. Insets show images taken at $\times 630$. The stippled line indicates the border between the luminal contents and the epithelial mucus layer. Panel 1: a WT Sham mouse; panel 2: a WT 9343-colonized mouse; panel 3: a WT 86-colonized mouse; panel 4: a Min $9343 \rightarrow 86$ co-colonized mouse. Strains 9343 and 86 colonize both the lumen and the mucosal surface with patchy mucus penetration (examples are shown with arrows) whether colonizing alone or in combination with another strain in the gut

a
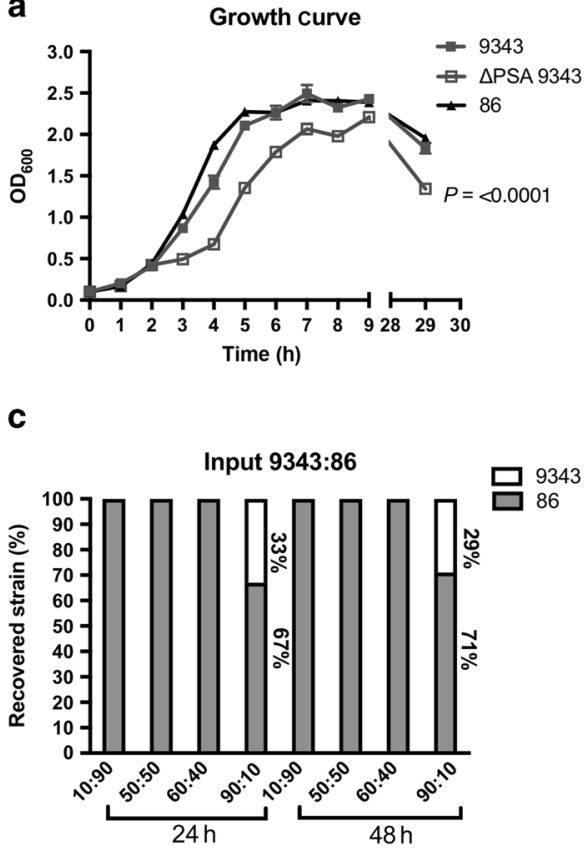

b

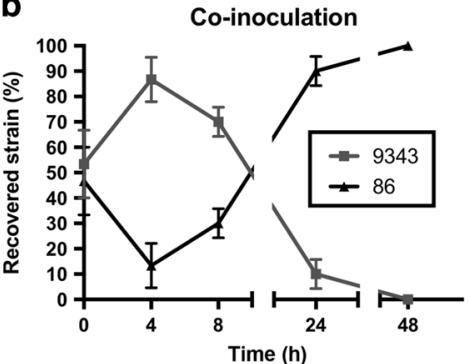

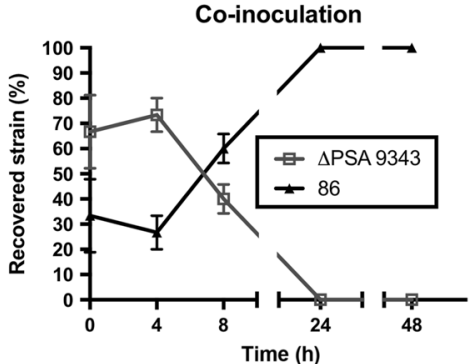

P-K $100^{\circ} \mathrm{C}$

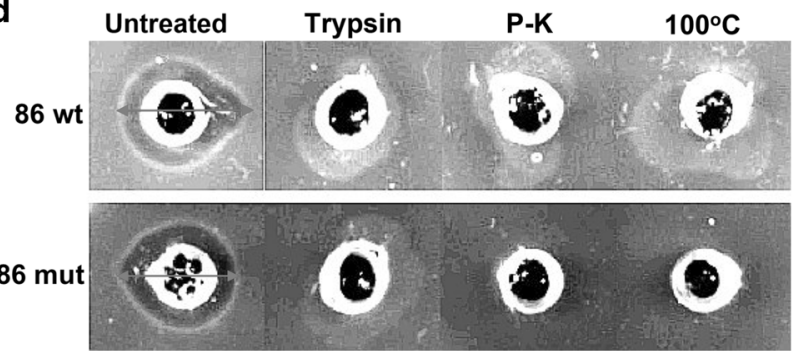

Fig. 5 The 86 strain secretes an intraspecies inhibitory molecule. a Individual 9343, $\triangle P S A$ 9343, and 86 growth curves. Curves represent the average of triplicate values. b Co-inoculation assay of 9343 with 86 and $\triangle$ PSA 9343 with 86 . Isolated B. fragilis colonies (10) plated from cocultures were randomly selected and screened by strain-specific PCR. After equal part inoculation, 9343 and $\triangle$ PSA 9343 are no longer detected at 24 and $48 \mathrm{~h}$. c Co-inoculation assay of 9343 with 86 . After differential inoculation, 9343 is only detected at an input ratio of $90: 10$ (9343:86) at 24 and $48 \mathrm{~h}$. d Agar diffusion assay of 9343 indicator strain (grown on the agar) with 86 supernatant (in wells). A zone of clearing (span of arrow) is induced by the 86 supernatant. Protease treatment with trypsin and proteinase- $\mathrm{K}$ and heat treatment at $100{ }^{\circ} \mathrm{C}$ impairs 86 supernatant activity. Top row: 86 wild-type (wt) supernatant; bottom row: 86 BFT mutant (mut) supernatant

regulation was similar between both strains in vivo (S9B Fig). This result does not rule out potential differential gene expression occurring when competing strains are both in contact in the murine host.

Therapeutic treatment with 9343 does not improve the health of 86-colonized mice

To further assess whether 9343 could abrogate disease therapeutically, SPF WT mice were stably infected with 86 for 2 weeks and then gavaged with 9343 daily, for 9 days $\left(86 \rightarrow 9343^{R}\right.$ ) (S1 Fig, probiotic protocol). Mice were killed after 2 days of rest from 9343 probiotic treatment. In a subset of $86 \rightarrow 9343^{R}$ mice ( 3 out of 5), we were able to introduce low-level stable colonization of 9343 with therapeutic treatment (Fig. 6a, diamonds-mouse ID: 62, 61, 59). Interestingly, low-level 9343 integration into stably colonized 86 mice led to no impact seen on 86 colonization or clinicopathology. Namely, $86 \rightarrow 9343^{R}$ mice continued to exhibit small, contracted ceca containing blood clots and diminished cecal content, a response to cecal injury, comparable to $86 \rightarrow \mathrm{PBS}^{\mathrm{R}}$ controls; cecum weights and splenomegaly (an indicator of chronic inflammation in our model) were indistinguishable between these groups
(Fig. 6b-d). Consistent with our results regarding the establishment of strain dominance, even with repeated exposure to 86, $9343 \rightarrow 86^{R}$ mice maintained the appearance of healthy ceca, displaying characteristic weight and content, with no spleen enlargement, comparable to Sham $\rightarrow \mathrm{PBS}^{\mathrm{R}}$ and $9343 \rightarrow \mathrm{PBS}^{\mathrm{R}}$ controls (Fig. $6 \mathrm{~b}-\mathrm{d}$ ). Although we noted that 86 had the capability to oust stably colonized 9343 in a few mice (Fig. 4c, e) resulting in marked tumorigenesis, in this series of experiments, we found that $9343 \rightarrow 86^{\mathrm{R}}$ mice ( 5 out of 5 ) did not develop stable 86 colonization (Fig. 6a, triangles). Overall, these data are consistent with the results of our colitis and tumor experiments, suggesting that 9343 may only be beneficial as a preventative measure against future 86 colonization.

The 9343 strain modulation of the immune system

Dependent on PSA, 9343 has been purported to promote immune tolerance through inducing the development of IL-10-producing Tregs. ${ }^{10,33}$ To further examine whether 9343 modulates immune responses in SPF mice, both SPF WT and SPF Min mice were colonized with 9343 or $\triangle \mathrm{PSA} 9343$ for 2 weeks. For this experiment, lamina propria lymphocytes (LPLs) were isolated 


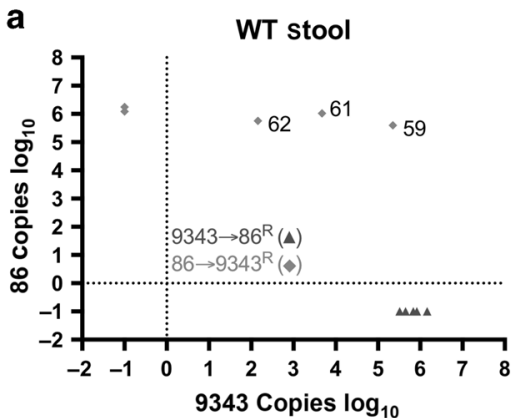

Mouse cecum $\&$ spleen (to scale)

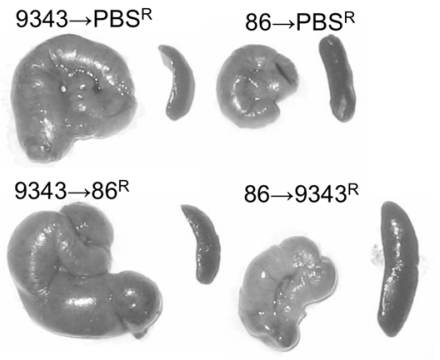

C

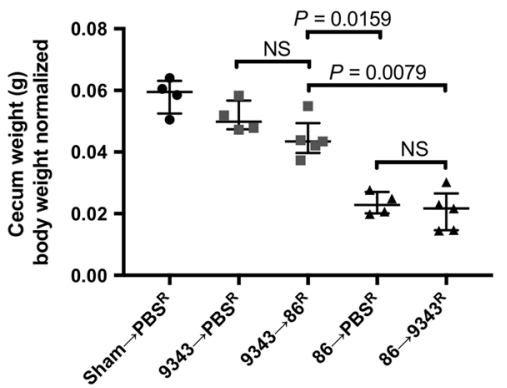

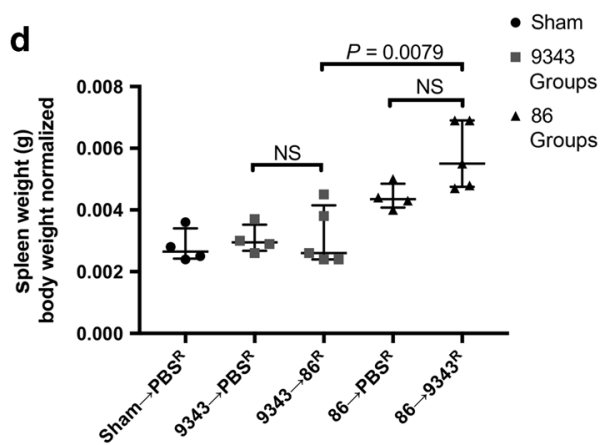

Fig. 6 Therapeutic treatment with 9343 does not improve the health of 86 -colonized mice. a-d SPF WT mice colonized with 86 or 9343 for 2 weeks were treated with 9343 or 86 , respectively $\left(86 \rightarrow 9343^{R}\right.$ and $\left.9343 \rightarrow 86^{R}\right)$. Superscript ${ }^{R}$ denotes repeated treatments for 9 days. Mice were rested for 2 days before killing. Data shown include a single experiment with 4 to 5 mice per group. Bars indicate median and interquartile range. a Strain quantification measured by stool qPCR at the time of killing. Symbols represent individual mice. Diamonds: 3 of 5 $86 \rightarrow 9343^{R}$ mice establish detectable levels $\left(10^{2}-10^{6}\right.$ copies) of treatment strain 9343 (mouse ID: 62, 61, 59). Triangles: 0 of $59343 \rightarrow 86^{R}$ mice

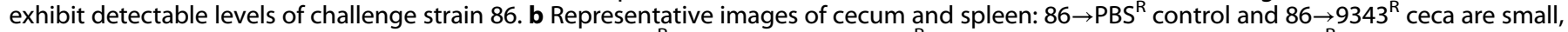
pale, and contracted; spleens are enlarged. The $9343 \rightarrow \mathrm{PBS}^{R}$ control and $9343 \rightarrow 86^{R}$ ceca are comparable to Sham $\rightarrow P^{2} S^{R}$ mice $($ not shown) in size, coloring, and content; spleens are not enlarged. c Mouse cecum weights. The $86 \rightarrow 9343^{R}$ cecum weights are comparable to $86 \rightarrow$ PBS $^{R}$ controls, indicative of disease. The $9343 \rightarrow 86^{R}$ cecum weights are higher compared to $86 \rightarrow P^{2} S^{R}$ control and $86 \rightarrow 9343^{R}$ mice, indicative of protection. d Mouse spleen weights. The $86 \rightarrow 9343^{R}$ spleen weights are higher compared to $9343 \rightarrow 86^{R}$ mice but comparable to $86 \rightarrow P B S^{R}$ controls, indicative of disease. The $9343 \rightarrow 86^{R}$ spleen weights are comparable to $9343 \rightarrow P B S^{R}$ controls, indicative of protection

from the distal colon, representing the colitis and tumor-prone locales $^{17}$ in the WT and Min ETBF 86 models, and studied by flow cytometry for immune cell composition. Strikingly, comparisons of 9343 and $\triangle \mathrm{PSA} 9343$ mice yielded unchanged IL-17A ${ }^{+}, \mathrm{FOXP3}^{+}$ (forkhead box P3), or IFN- $\gamma^{+}$T-cell populations as well as IL-17A ${ }^{+}$ $\mathrm{FOXP3}^{+}$populations whether analyzed by percentage or cell density (Fig. 7a, S10 Fig: filled circles $=$ WT; open circles $=$ Min). Though the colon LP represents the anatomical site most reflective of, and meaningful to, the immune responses initiated by intestinal microbial exposure, similar results were also obtained in the mesenteric lymph nodes (MLNs) and spleens (S11 Fig). These data were therefore consistent with the analysis of whole tissue transcriptional expression of $1 / 17 a, \| 110$, and Ifng genes in SPF WT mice (Fig. 1a).

Since the original work on systemic and mucosal PSA-induced immunomodulation had primarily focused on GNO mice, ${ }^{10,11,22}$ we further extended our investigation to GNO WT mice monocolonized with 9343 or $\triangle$ PSA 9343 for either 2 or 8 weeks. As 9343 had been reported to promote immune system maturation in the naive host, altering the architecture of splenic follicles, ${ }^{22,34}$ we first examined both the splenic morphology and histology of control and monocolonized GNO WT and matched single colonized SPF WT mice. Spleens from mice across all treatment groups and housing backgrounds exhibited a normal size and weight, and histological sections revealed normalappearing lymphoid development in the form of well-defined follicles comprising the white pulp (S12 Fig). Furthermore, surprisingly, flow cytometric analysis of the GNO colon LPLs yielded no differences in the accumulations of Th17, Treg, Th1, or total IL-17A ${ }^{+}$populations between 9343 and $\triangle P S A 9343$ monocolonized mice at early or late time points as reflected by absolute cell number (cell density) (Fig. 7b). In parallel, we analyzed the transcription of functionally relevant cytokines to the described immune cell populations. We did not detect any differences in stereotypical Th17 or Treg cytokines, $1 / 17 a$ and II10, in 9343 versus $\triangle$ PSA 9343-treated mice; however, we did detect increased I/17a mRNA expression in monocolonized GNO mice compared with uninfected germ-free (GF) controls at 2 but not at 8 weeks. No differences in the expression of characteristic Th1 and Th2 cytokines, Ifng and //4, were observed among groups (Fig. 7c). Accumulations in IL-17A-producing or IFN- $\gamma$ producing $\gamma \delta \mathrm{T}$ cells were also comparable between groups (S13 Fig). However, the total IFN- $\gamma$-producing cell population was significantly less in GNO mice colonized with $\triangle$ PSA 9343 for 2 weeks and then significantly greater at 8 weeks when compared to PSA-sufficient 9343 (Fig. 7b), suggesting a possible role of PSA in regulating IFN- $\gamma$-producing cells in the GNO host. However, this result was not confirmed by whole tissue gene expression analysis (Fig. 7c). Taken together, in our hands, colonization with PSA-sufficient 9343 did not modulate SPF (WT or Min) or GNO (WT) host immune responses differently than colonization with PSA-deficient 9343.

\section{DISCUSSION}

To discern the probiotic potential of NTBF 9343 in gut health, we studied how 9343 impacted an ETBF 86-driven disease model of colitis and tumorigenesis. SPF C57BL/6 WT and $\mathrm{Min}^{\text {Apc716+/- }}$ mice were used in a $B$. fragilis co-infection model where mice were sequentially or simultaneously treated. We showed that 9343 
a
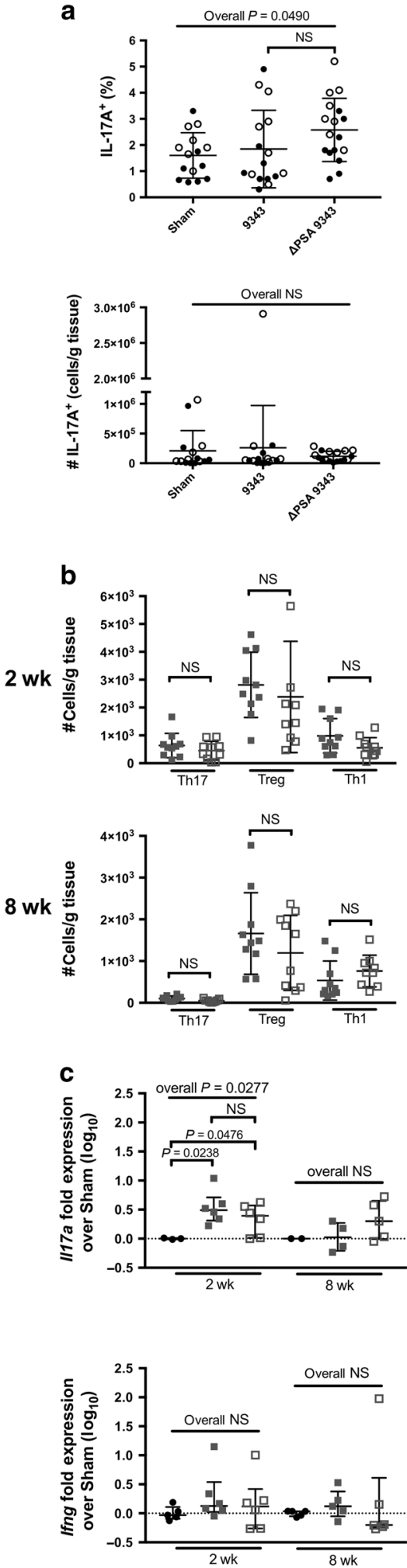

limited 86 disease severity, significantly reducing the IL-17Amediated inflammatory signature and reducing the development of colon tumors. However, this finding was limited to sequentially infected mice, receiving 9343 then 863 days later $(9343 \rightarrow 86)$, but, nonetheless, suggesting the potential of 9343 prophylaxis. In contrast, simultaneously treated $(9343+86)$ or sequentially treated mice, 86 then 93433 days later $(86 \rightarrow 9343)$, continued to exhibit
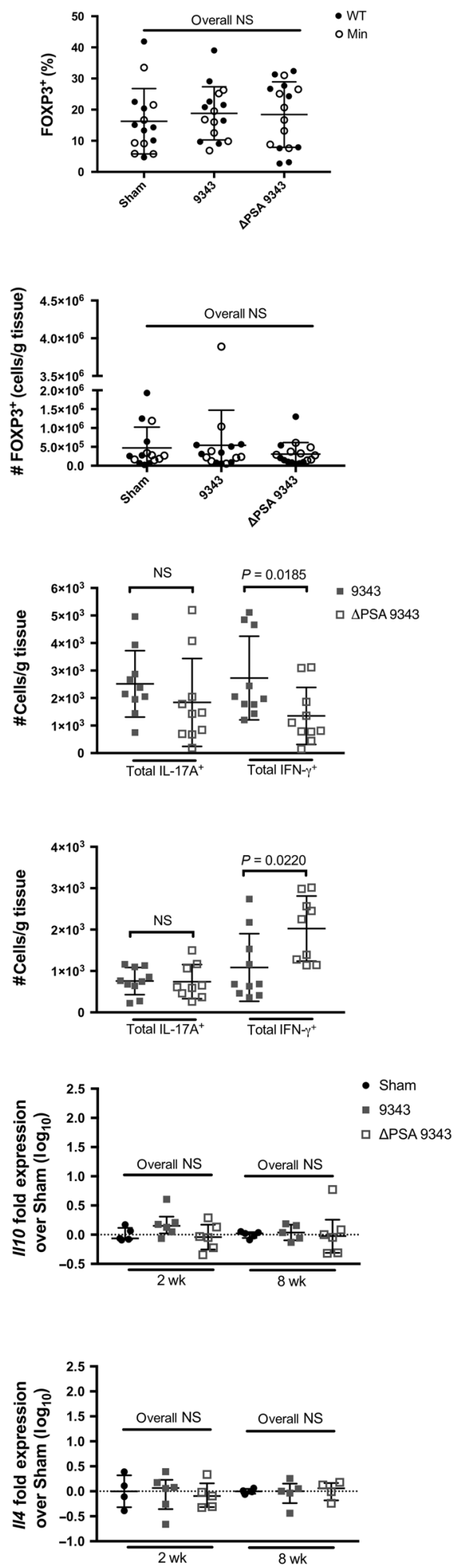

severe disease, even when 9343 colonized with comparable copy numbers as 86 (Fig. 4b-e). Notably, although prophylactic 9343 reduced pro-inflammatory $1 / 17 a$ gene expression, it failed to fully block IL-17A in the colitis setting (Fig. 1a). Consistent with this finding, we observed the development of microadenomas in $9343 \rightarrow 86$ mice that did not progress to visible macroadenomas, even after 16 weeks (Fig. 2) (S4 Fig). Previous studies in 
Fig. 7 Analysis of immune responses to PSA-competent and PSA-deficient 9343 colonization. a LPLs were isolated from distal colons of SPF WT and SPF Min mice 2 weeks after Sham, 9343, or $\triangle$ PSA 9343 treatment and subjected to 4-h incubation with cell stimulation cocktail and subsequent intracellular cytokine staining (ICS) for IL-17A and FOXP3. Data are presented as percent IL-17A ${ }^{+}$of live CD3 ${ }^{+}$lymphocytes and percent $\mathrm{FOXP3}^{+}$of live $\mathrm{CD}^{+} \mathrm{CD}^{+}$lymphocytes (top). $\mathrm{CD}^{+} \mathrm{IL}^{-17 \mathrm{~A}^{+}}$and $\mathrm{CD}^{+} \mathrm{CD}^{+}$Foxp3 $^{+}$cell numbers were also normalized by colon tissue mass (bottom). Bars indicate mean \pm 1 SD. Data shown include 2 replicate experiments, each 4 to 5 mice per group. No differences were noted among 9343 and $\triangle$ PSA 9343 groups in the parameters tested. b LPLs were isolated from distal colons of GNO WT mice 2 and 8 weeks after Sham (remained GF), 9343, or $\triangle$ PSA 9343 treatment. Cells were stimulated and subsequent ICS was performed. Data represent absolute cell number normalized to colon tissue mass. Bars indicate mean \pm 1 SD. Data shown include 2 replicate experiments, each 4 to 5 mice per group. Only total IFN- $\gamma^{+}$results differed between 9343 and $\triangle$ PSA 9343 mice. c In parallel, GNO WT mouse distal colon tissue was harvested for whole tissue qRT-PCR. Bars indicate median and interquartile range. Data shown include 2 replicate experiments, each 2 to 3 mice per group. No differences were observed among groups in total II10, Ifng, and I/4 expression, although monocolonized (9343 and $\triangle$ PSA 9343) mice exhibited increased $I / 17 a$ expression compared with uninfected GF controls (Sham) at 2 weeks but not at 8 weeks. Multiple group comparisons were conducted using Kruskal-Wallis testing and pairwise comparisons using Mann-Whitney $U$ testing

SPF recombination activating gene (Rag)-deficient mice showed that 9343 or purified PSA was protective against CD45RB ${ }^{\text {high }}$ T-cell transfer/H. hepaticus-induced colitis, whereas $\triangle \mathrm{PSA} 9343$ was not. ${ }^{21}$ Purified PSA administration also protected SPF mice from TNBS chemically induced colitis. ${ }^{10,21}$ Though our results show that 9343 partially subverted both 86-induced colitis and tumorigenesis, most surprisingly, we discovered this effect was independent of 9343-encoded PSA. Namely, we observed similar 86 disease prevention in mice previously colonized with the 9343 parental strain as well as in mice previously colonized with the PSAdeficient 9343 strain.

It is possible that the resident microbiota may temper the effects of colitis. For instance, 86 induces rapid lethality in GNO mice, $^{13}$ while GNO mice colonized with 9343 prior to 86 exposure exhibit longer survival ( $\mathrm{S}$. Wu et al. unpublished data). The 9343 colonization of GNO mice also ameliorated dextran sulfate sodium (DSS)-induced colitis. ${ }^{35}$ Similarly, our study found that 9343 mitigated colitis and tumorigenesis initiated by 86 , as long as 9343 introduction both preceded 86 exposure and remained the dominant B. fragilis strain. Thus, we hypothesize that 9343 colonization in the host can act as a barrier, either through niche exclusion or secreted factors, to certain colitogenic factors in the gut (e.g., ETBF 86 and DSS). In our model, the pattern of colonization burden in $9343 \rightarrow 86$ sequentially treated mice showed clear evidence of 9343 competitive exclusion of 86 , likely diminishing epithelial cell damage or bacterial invasion that could be induced by the latter. In contrast, $9343+86$ simultaneously treated mice typically exhibit competitive exclusion of 9343, precluding any defense against 86 disease. A prior report indicated that $B$. fragilis species colonization resistance may be absolute, ${ }^{24}$ using differentially labeled 9343 isogenic strains in GNO mice. In contrast, using a co-infection protocol with nonisogenic strains (9343 and 86) in SPF mice, we found that $B$. fragilis competitive exclusion was not absolute and, in fact, a sizeable minority of $9343 \rightarrow 86$ mice, $86 \rightarrow 9343$ mice, and $9343+86$ mice displayed high level $\left(\geq 10^{5}\right.$ bacterial copies/100 ng) fecal cocolonization (S1 and S2 Tables). Select strain-specific colon mucosal niches for 9343 and 86 were not detected in these mice (Fig. 4f, panel 4). Persistent colonization (up to 4 months) with a $B$. fragilis challenge strain was consistently observed in our mice, with even a few instances of complete 9343 displacement by 86, observed in $9343 \rightarrow 86$ mice (Fig. $4 \mathrm{~b}-\mathrm{e}$ ). Thus, our results suggest that co-colonization with non-isogenic $B$. fragilis strains is possible. Lastly, prior results ${ }^{11}$ suggested that PSA-insufficient 9343 colonized the mucosa of GNO mice less well than PSA-sufficient 9343, whereas herein we identified similar mucosal colonization for both strains in conventional and GNO mice (Fig. 3b and S6). Differences in approach and quantification of mucosal adherence between studies likely account for these observations.

Bacterial fitness factors may also contribute to the establishment of strain dominance. Despite impaired growth observed in the absence of PSA, the monitoring of WT 9343 and WT 86 in vitro growth (Fig. 5a) did not suggest differences in nutrient utilization, nor was differential regulation of colonization factors observed in vivo (S9B Fig). Yet, we did observe support for 86 secretion of a bacteroicin-like molecule, easily harvested from culture supernatants and inhibitory to 9343 growth, independent of BFT (Fig. $5 d$ ). Due to evidence of transcription of the BSAP-1 gene (BF638R_1646) by 86 in vitro (S9A Fig), we suggest that BSAP-1, a pore-forming molecule, is a likely candidate in the broad inhibitory action of 86 on other strains contributing to its own survival during simultaneous co-infection with $9343(9343+86)$. Furthermore, type VI secretion (T6S), the direct injection of effector proteins into the membrane of a target organism, has been implicated in the maintenance of bacterial competition. For instance, the $B$. fragilis T6S system demonstrates effectiveness against closely related Bacteroides thetaiotaomicron and other $B$. fragilis strains. ${ }^{36,37}$ The $B$. fragilis T6S effector-immunity region, tssC, was found to shape intraspecies competition; parental NTBF 9343 protected SPF mice from an ETBF (strain ATCC43858) DSSmediated colitis through lowering ETBF strain colonization, whereas a 9343 tssC mutant did not. ${ }^{38}$ In our model, T6S may be a way that 9343 precludes 86 disease during sequential treatment $(9343 \rightarrow 86)$. We hypothesize that both timing and order of colonization and secretion of inhibitory molecules contribute to $B$. fragilis competition dynamics.

ETBF strain carriage in healthy, asymptomatic people is quite common with a recent study showing that up to $40 \%$ of individuals ( 6 out of 15 subjects) in Boston, MA, carried ETBF in their feces. ${ }^{39}$ Yet, clinically, ETBF is known as an etiologic agent of acute diarrhea in children and adults and is also highly associated with active IBD and CRC. ${ }^{18,40,41}$ Thus, we evaluated the possible remedial effect of NTBF 9343 treatment on pre-existing ETBF 86 disease. Therapeutic treatments of stably colonized 86 mice with $9343\left(86 \rightarrow 9343^{R}\right)$, failed to abrogate disease severity. However, we did show evidence of 9343 strain persistence in a subset of mice (3 out of 5) (Fig. 6), again confirming that B. fragilis strain cocolonization in vivo is not infrequent, as a prior report suggested. ${ }^{24}$ In human fecal microbial transplantation, variable and incomplete uptake of recipient strains has also been reported. ${ }^{42}$ Such findings stress the importance of longitudinal clinical studies coupled with bioinformatics approaches to understand probiotic-recipient or donor-recipient compatibility and their impact on pre-existing disease states.

Collectively, our data demonstrate that the absence of PSA neither diminishes the protective capability of NTBF strain 9343 nor primes SPF mice for inflammatory Th17 polarization. Specifically, we demonstrated that SPF mice singly colonized with 9343 or $\triangle P S A 9343$ displayed neither an inherent difference in inflammatory or regulatory T-cell levels in the LP (IL-17A ${ }^{+}, \mathrm{IFN}-\mathrm{\gamma}^{+}$, $\mathrm{FOXP3}^{+}$) (Fig. 7) (S10 Fig) nor differences in transcriptional expression of colon tissue cytokines (IL-17A, IFN- $\gamma$, IL-10) (Fig. 1a). These data contrast with reports of $\mathrm{FOXP3}^{+}$Treg cell accumulation and IL-10 production in response to oral PSA treatment in SPFhoused mice. ${ }^{10,21}$ It also contrast with reports of increased IFN- $\gamma$ production from $\mathrm{CD}^{+} \mathrm{T}$ cells in GNO mice monocolonized with 
174

9343..$^{22}$ Importantly, both histopathologic colitis and tumor development was similarly limited by both PSA-sufficient and PSA-deficient 9343 strains (Figs. 1 and 2). Geis et al. ${ }^{43}$ recently described a mechanism by which $\mathrm{FOXP3}^{+}$Treg cells initiated the inflammatory Th17 response during 86 infection. Thus, we also analyzed the accumulation of IL-17A ${ }^{+} \mathrm{FOXP3}^{+} \mathrm{T}$ cells and found no differences among groups (S10 Fig).

To study 9343 and $\triangle$ PSA 9343 in isolation, we also used GNO mice, similar to prior reports. ${ }^{10,11,21,22}$ Contrasting with earlier reports, ${ }^{22,34}$ we did not observe PSA inducement of lymphoid organogenesis in the spleen (S12 Fig). Furthermore, to our surprise, PSA-competent 9343 did not affect the accumulation of LP Treg or Th1 cells, nor differ from $\triangle$ PSA 9343-colonized mice in the accumulation of Th17 cells, at early ( 2 week) or late ( 8 week) time points (Fig. 7b). Unexpectedly, increased $1 / 17 a$ expression was observed in monocolonized (9343 or $\triangle P S A$ 9343) GNO mice compared with uninfected GF controls at 2 weeks, suggesting that $B$. fragilis colonization alters the GNO immune system early, but parallel assessment of $1 / 10$, Ifng, and $/ 14$ yielded no differences among groups (Fig. 7c). There were also no differences seen in the recruitment of IFN- $\gamma$-producing or IL-17A-producing $\gamma \delta$ T cells (S13 Fig). Although we observed increased accumulation in total IFN- $\gamma$ producing cells in $\triangle$ PSA 9343 mice at 8 weeks compared to 9343 mice (Fig. 7b), this finding contrasted with previous reports describing the reverse, where PSA-competent 9343 induced IFN- $\gamma$ and reduced IL-4 production in stimulated splenic T cells. ${ }^{22}$ Finally, $\triangle$ PSA 9343-colonized SPF and GNO mice did not exhibit diminished mucosal colonization levels, contrasting with a previous study, ${ }^{11}$ nor did the mutant strain exhibit differential localization (Fig. 3). A key feature of our findings is that they represent the analysis of cells and tissues isolated from the colons of GNO and SPF mice on the C57BL/ 6 background. The earliest discoveries reporting 9343-directed immune system maturation, lymphoid development, and niche occupation were made in GNO Swiss-Webster mice obtained from Taconic. ${ }^{21,22,24,25}$

We demonstrate that NTBF strain 9343 offers a preventative health benefit to the host, not reliant on polysaccharide $A$ or on direct host immune modulation. Interestingly, cultures of mucosal biopsies revealed that PSA-sufficient human isolates of $B$. fragilis are not found more frequently at non-inflamed versus inflamed sites in IBD patients (Crohn's disease, $p=0.68$; ulcerative colitis, $p$ $=1.0$ ), nor are PSA-sufficient strains more often detected in healthy controls versus IBD patients $(p=0.73){ }^{44}$ Thus, in the available human data, PSA is not yet associated with colon mucosal health. Our data indicate that the order in which the host sees 9343 or 86 , the specific $B$. fragilis strains being introduced to and already existing in the recipient, and bacterial fitness are crucial to determining overall probiotic efficacy.

Numerous Bacteroides clinical isolates displayed bacteriocin production and sensitivity. ${ }^{45,46}$ These diverse responses point to the complexity of microbial niche establishment, also laying the groundwork for probiotic engineering that may exploit advantageous fitness factors. Similar to an oral bacterial strain replacement therapy devised to prevent dental cavities caused by acidic Streptococcus, ${ }^{47}$ specific intestinal isolates, such as 9343 , could be engineered to outcompete and replace pathogenic gut bacteria, possibly through potent bacteriocin production. Recently, the administration of non-toxigenic Clostridium difficile spores significantly reduced the recurrence of toxigenic $C$. difficile infection in a randomized clinical trial, remaining well tolerated and safe in patients. ${ }^{48}$ In diseases with protracted etiology, such as CRC, the introduction of beneficial or non-pathogenic bacterial species or strains may contribute to improved clinical outcomes over the long term, possibly through limiting the oncogenic potential of individual microbes. ${ }^{14,19}$ Our findings, describing conditional NTBF 9343 protection from ETBF 86 pathogenesis, stress the importance of understanding the complex interactions of intestinal microbes with one another (microbe-to-microbe), down to the strain level, rather than to only focus on the interactions of intestinal microbes with their host (microbe-to-host). Further research is needed to better understand microbial interaction specificity, the effects of single microbes on the entire microbial community, and the factors contributing to the establishment of a stable microbiome. These features will be vital in the selection of optimal probiotic bacterial strains.

\section{METHODS}

Bacterial strains and growth

B. fragilis strains NCTC 9343 (9343), ${ }^{13,14}$ NCTC $\triangle$ PSA 9343 ( $\triangle$ PSA 9343) (isogenic mutant), ${ }^{49}$ and $86-5443-2-2(86)^{13,14}$ were used for experiments except as indicated. B. fragilis strains VPI 13784 (ETBF, BFT-1) and Korea 570 (ETBF, BFT-3) were utilized for agar diffusion assays (S8 Fig). All strains were clindamycin resistant: ETBF 86 strains (naturally) and NTBF 9343 strains (pFD340-transformed resistance). Strains were grown anaerobically at $37^{\circ} \mathrm{C}$ with brain-heart infusion ( $37 \mathrm{~g} / \mathrm{L} ; \mathrm{BD}$ Bacto) liquid media or agar plates (1.5\% BD Bacto Agar) supplemented with yeast extract $(5 \mathrm{~g} / \mathrm{L} ; \mathrm{BD}$ Bacto), L-cysteine (50 mg/L; Sigma), hemin ( $0.5 \mathrm{mg} / \mathrm{L}$; Sigma), and vitamin K (0.1 mg/L; Sigma). Glycerol stocks of 9343 and 86 were cultured on $\mathrm{BHI}$ clindamycin $(10 \mu \mathrm{g} / \mathrm{L}$; Research Products International) plates and isolated colonies sub-cultured in liquid media for $\sim 24 \mathrm{~h}$. For mouse inoculums, bacterial pellets were washed and resuspended with $1 \times$ Dulbecco's phosphate buffered saline $(1 \times$ PBS free of calcium chloride and magnesium chloride).

For growth curves, strains were grown to mid-logarithmic phase, diluted to an $\mathrm{OD}_{600}$ of 0.1 in a pre-warmed, pre-reduced culture tube (BHI clindamycin), and monitored every hour for $9 \mathrm{~h}$ and then at $29 \mathrm{~h}$. Bacterial turbidity was monitored by spectrophotometer $(600 \mathrm{~nm})$ and subcultures performed (48 $\mathrm{h}$ incubation) to quantify viable CFUs per $\mathrm{mL}$. For in vitro co-inoculation assays, 9343 and 86 were grown to mid-logarithmic phase and either introduced to a fresh culture tube (BHI clindamycin) equally or at varying ratios (9343 to 86: roughly 10:90, 50:50, 60:40, 90:10), and cultured up to $48 \mathrm{~h}$. At sampling, subcultures were performed to allow random selection of 10 colonies tested for colony identification (9343 vs 86) using strain-specific PCR (S3 Table). ${ }^{18,50}$ Percentages of 9343- and 86-positive colonies were determined. All assays were run in triplicate.

For agar diffusion assays, 9343 and 86 strains were grown separately in BHI media (no antibiotics). After $48 \mathrm{~h}$, bacterial suspensions were centrifuged $(13,000 \mathrm{rpm}, 5 \mathrm{~min})$ and culture supernatants sterile-filtered $(0.22 \mu \mathrm{m})$. Then, $500 \mu \mathrm{L}$ of the indicator strain, 9343, was mixed with $12 \mathrm{~mL}$ of $\mathrm{BHI}$ soft agar $(0.7 \%)$; after drying, hole punches $(6 \mathrm{~mm})$ were created, sealed with $35 \mu \mathrm{L}$ of soft agar, and inoculated with $100 \mu \mathrm{L}$ of supernatant. Plates were grown anaerobically overnight and observed for an inhibition zone. Supernatant treatments included trypsin $(200 \mu \mathrm{g} /$ $\left.\mathrm{mL}, 2 \mathrm{~h}, 37^{\circ} \mathrm{C}\right)$, proteinase- $\mathrm{K}\left(100 \mu \mathrm{g} / \mathrm{mL}, 2 \mathrm{~h}, 37^{\circ} \mathrm{C}\right)$, and heat inactivation $\left(15 \mathrm{~min}, 100^{\circ} \mathrm{C}\right)$.

Mice and infection model

SPF C57BL/6 WT mice (obtained from Jackson Laboratories and maintained in house) and C57BL/6 multiple intestinal neoplasia $\left(\right.$ Min $\left.^{A p c 716+/-}\right)$ mice (obtained from Dr. David Huso, Johns Hopkins University) were used for all experiments. Mice, 3 to 4 weeks old, were placed on antibiotic water bottles (clindamycin $0.1 \mathrm{~g} / \mathrm{L}$ and streptomycin $5 \mathrm{~g} / \mathrm{L}$ ) (Hospira and Amresco) for 5 to 7 days and discontinued. Each experiment included mice of both sexes. No experimental differences among mice of differing sex were identified (not shown). Mice were then gavaged with $\sim 10^{8}$ CFUs of 9343, $\triangle P S A$ 9343, and/or 86; Sham controls were gavaged with $1 \times$ PBS. Treatment groups included: (1) colonization with one strain $(9343, \triangle P S A$ 9343, or 86), (2) sequential treatment with initial strain at day 0 and challenge strain at day $3(9343 \rightarrow 86, \triangle P S A$ $9343 \rightarrow 86$, or $86 \rightarrow 9343$ ), and (3) simultaneous treatment with both 
strains $(9343+86)$. Fecal colonization of the initial strain was confirmed for sequentially treated mice prior to challenge strain administration. For co-infection colitis experiments, mice were killed 2 weeks after infection with the initial bacterial strain in WT mice, at 2 to 4 weeks in Min mice for the microadenoma experiments, and at 12 weeks in Min mice for the macroadenoma experiments. For GNO colonization experiments, C57BL/6 GF WT mice were gavaged at 6 to 10 weeks old and killed at both 2 and 8 weeks post colonization.

For therapeutic treatment experiments, SPF WT co-infected mice were colonized with a single strain for 2 weeks ( 86 or 9343) and fecal colonization confirmed. Mice were gavaged with 9343 (when pre-colonized with 86 ) or 86 (when pre-colonized with 9343) daily, for 9 days. Control 9343 or 86 -only mice were gavaged with $1 \times$ PBS. Mice were rested for 2 days and killed at 24 days after initial strain administration. The Johns Hopkins University Animal Care and Use Committee approved all experimental protocols. Mice were held in SPF conditions in accordance with the Association for the Assessment and Accreditation of Laboratory Animal Care International. GF mice were bred and maintained in the Johns Hopkins University GF mouse facility.

Fecal colonization and mucosal adherence

Mouse stools were weighed, homogenized $\left(10^{-1}\right.$ dilution), and diluted $\left(10^{-5}, 10^{-6}, 10^{-7}\right)$ in $1 \times$ PBS. At the time of killing, mouse distal colon tissue $(\sim 1 \mathrm{~cm}$ in length) was harvested, weighed, and washed twice (30 s, vortex setting 7 ) with $0.016 \%$ dithiothreitol (Thermo Scientific) in $1 \mathrm{~mL}$ of $1 \times$ PBS to quantitate tightly adherent mucosal bacteria. The tissue was transferred to $350 \mu \mathrm{L}$ $1 \times$ PBS, homogenized, and diluted $\left(10^{-1}, 10^{-2}, 10^{-3}\right)$. Dilutions of stool and colon tissue were plated onto $\mathrm{BHI}$ clindamycin agar and grown anaerobically for $\sim 48 \mathrm{~h}$ for determination of viable bacterial CFUs. Data are presented as $B$. fragilis CFUs per gram of stool or tissue.

\section{Quantitative RT-PCR}

Distal colon tissue RNA was extracted using the Qiagen RNeasy Mini Kit according to the manufacturer's instructions. RNA $(1 \mu \mathrm{g})$ was reverse transcribed to complementary DNA using the Superscript III Reverse Transcriptase kit (Invitrogen). For each sample, two technical replicates were run with Taqman Gene Expression Mix. According to standard amplification protocols, samples were run on the 7500 Real-Time PCR System (Applied Biosystems) for 40 cycles. Cytokine primer/probe sets were purchased from Applied Biosystems. Relative quantification $(\Delta \Delta \mathrm{Ct})$ of indicated genes was determined by subtraction of endogenous control 185 rRNA and then subtraction of normalized uninfected control (Sham) values from colonized test values. Fold change was determined by $2^{-\Delta \Delta \mathrm{Ct}}$.

Fecal DNA was extracted using the Fast Stool DNA Kit (Qiagen) according to the manufacturer's instructions. Using the Taqman system, supplemented with bovine serum albumin, $100 \mathrm{ng}$ of stool DNA was measured with three technical replicates per sample for 40 cycles (AB 7500). Multiplex bft/16S qPCRs ${ }^{18,51}$ were run at $58^{\circ} \mathrm{C}$; singleplex $9343-\mathrm{Tn}$ qPCRs were run at $59^{\circ} \mathrm{C}$ (S3 Table). ${ }^{50}$ Bacterial strain quantification was determined by standard curve from serially diluted $\left(10^{-1}\right) 9343$ and 86 genomic DNA.

\section{Histology, microadenoma, and macroadenoma counts}

Colons were dissected, Swiss rolled, and fixed with $10 \%$ buffered formalin for $\sim 24 \mathrm{~h}$. Histology examination and scoring was done on hemotoxylin and eosin (H\&E)-stained $5 \mu \mathrm{m}$ sections. Disease scoring was done as previously described ${ }^{14}$ by a veterinary pathologist (Dr. Sarah Beck). Swiss rolled H\&E-stained sections of

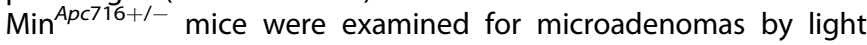
microscope at $\times 200$ total magnification. At late time points, $\mathrm{Min}^{\mathrm{ApC} 716+/-}$ colons were flushed, opened, fixed with formalin for $\sim 24 \mathrm{~h}$, and stained with methylene blue solution ( $0.2 \%$ in saline) (Sigma) for $1 \mathrm{~h}$. Macroadenomas were counted using a dissecting scope (Leica ES2).

Immunofluorescence (IF) and FISH staining Unflushed mouse colons were fixed in formalin for $24 \mathrm{~h}$, suspended in saline, and paraffin-embedded (FFPE). For both staining protocols, tissue sections were stained with Periodic acid-Schiff to confirm mucus preservation (S5 Fig). Deparaffinized histological sections $(5 \mu \mathrm{m})$ were subjected to a standard IF protocol as follows: (1) initial block with $1 \times$ PBS ( $5 \%$ goat serum, $1 \%$ bovine serum albumin) for $1 \mathrm{~h}$ at room temperature (RT), (2) incubation overnight at $4{ }^{\circ} \mathrm{C}$ with primary antibodies: adsorbed 9343 rabbit anti-serum (1:100) and adsorbed 86 rat anti-serum (1:100), (3) two washes, $1 \times$ PBS, (4) incubation at RT for $1 \mathrm{~h}$ with secondary antibodies: goat anti-rabbit AF488 (1:100) (Sigma, A11034) and goat anti-rat AF568 (1:100) (Sigma, A-1107), (5) two washes, $1 \times$ PBS, (6) incubation with 4',6-diamidino-2-phenylindole (DAPI) $5 \mathrm{~min}$, (7) two washes, $\mathrm{dH}_{2} \mathrm{O}$, (8) mounted with ProLong Gold Antifade (Invitrogen) reagent. To limit cross-reactivity, 9343specific rabbit serum was adsorbed to uninfected SPF mouse stool and both live and formalin-fixed 86 and E. coli; 86-specific rat serum was adsorbed to uninfected SPF mouse stool and both live and formalin-fixed 9343 and E. coli (S7 Fig).

FFPE colon sections $(5 \mu \mathrm{m})$ were hybridized with a $B$. fragilis bacterial probe (IDT) (S3 Table) $)^{52}$ and stained with DAPI. Probes were applied at a concentration of $2 \mu \mathrm{M}$ in pre-warmed hybridization buffer $(900 \mathrm{mM} \mathrm{NaCl}, 20 \mathrm{mM}$ Tris $\mathrm{pH} 7.5,0.01 \%$ SDS). Slides were incubated at $46^{\circ} \mathrm{C}$ for $2 \mathrm{~h}$. Slides were washed three times for $5 \mathrm{~min}$ with FISH wash buffer $(215 \mathrm{mM} \mathrm{NaCl}, 20 \mathrm{mM}$ Tris pH 7.5, 5 mM EDTA). Coverslips were mounted with ProLong Gold Antifade (Invitrogen) reagent. All slides were imaged with the Zeiss LSM 780 confocal microscope.

$\mathrm{LPL}$, spleen, and MLN isolations

Dissected distal colons (defined as everything distal of the proximal colon striated or feathered architecture) were flushed. Colons were cut to $<0.5 \mathrm{~mm}$ pieces and washed in $1 \times$ sodium bicarbonate buffer supplemented with EDTA $(2 \mathrm{mM})$, fetal calf serum (FCS; 10\%), HEPES $(25 \mathrm{mM})$, and Hanks' balanced salt solution (HBSS) for $20 \mathrm{~min}$ at $37^{\circ} \mathrm{C}$, three times. Tissues were digested in RPMI supplemented with FCS (5\%), liberase (400 units/ $\mathrm{mL}$; Roche Diagnostic), and DNAse $1(0.2 \mathrm{mg} / \mathrm{mL}$; Roche Diagnostic) for $30 \mathrm{~min}$. Percoll gradient $(20 \%, 40 \%, 80 \%)$ (GE Healthcare Life Science) separation was used to isolate mononuclear cells. Harvested spleens and mesenteric lymph nodes were each pooled and digested in RPMI ( $5 \%$ FCS), with liberase and DNAse 1 for $30 \mathrm{~min}$. Accu-Prep (Accurate Chemical) cell separation media were used to isolate mononuclear cells.

\section{Flow cytometry}

For SPF and GNO experiments, LPLs from individual colons, 4 to 5 mice per group, were isolated. Isolated mononuclear cells were cultured in Iscove's Modified Dulbecco's Medium (IMDM) supplemented with FCS (5\%) with Cell Stimulation Cocktail (containing protein transport inhibitors) (eBioscience) in 96-well plates for $4 \mathrm{~h}$. Cells were washed with $1 \times$ PBS, stained for cell surface markers, fixed, and permeabilized. ICS was done for IL-17A, IFN- $\gamma$, and FOXP3. Flow cytometry was performed with the BD LSRII. Data were analyzed with BD FACSDiva 6.1.3 software.

\section{Statistical analysis}

Data were analyzed using the nonparametric Kruskal-Wallis test for multiple group comparisons. For multiple group comparisons achieving statistical significance with Kruskal-Wallis testing, we then performed subsequent two group comparisons of biological interest using the nonparametric two-tailed Mann-Whitney U-test. A $p$ value of $<0.05$ was denoted as significant. For correlation 
analysis, the nonparametric two-tailed Spearman's test was used. An rs value of $\geq 0.7$ was considered a strong positive correlation. Repeated measures analysis of variance was used for bacterial growth curve analysis.

\section{ACKNOWLEDGEMENTS}

The authors would like to thank the members of the Sears laboratory for their valuable, thoughtful discussion and comments regarding this work. The funding for this work was provided by the Bloomberg Philanthropies and the National Institutes of Health. The grant numbers covered under these funders include the following: R01DK080817 (to C.L.S.), R01CA151325 (to C.L.S. and D.M.P.), P30DK089502 (Gastrointestinal Center Grant), P30CA006973 (Sidney Kimmel Comprehensive Cancer Center core), P50CA062924 (Gastrointestinal Specialized Programs of Research Excellence), and S10OD016374 (JHU SOM Microscope Facility). The funders had no role in study design, data collection and analysis, decision to publish, or preparation of the manuscript.

\section{AUTHOR CONTRIBUTIONS}

Conception and design: J.L.C., S.W., and C.L.S. Development of methodology: J.L.C., S. W., F.H., and C.L.S. Acquisition of data (experimental and animal assistance, provided animals, provided facilities, etc.): G.V.C., T.A.M.G., H.F., X.W., H.D., D.L.H., D.M.P., F.H., and C.L.S. Analysis and interpretation of data (e.g., histopathological scoring, tumor counting, experimental analyses): J.L.C., S.W., S.E.B., H.W., F.H., and C.L.S. Writing, review, and/or revision of the manuscript: all authors. Administrative, technical, or material support (i.e., reporting or organizing data, flow cytometry analysis): J.L.C., S. W., A.L.G., A.J.T., L.C., and F.H. Study supervision: F.H. and C.L.S.

\section{ADDITIONAL INFORMATION}

The online version of this article (https://doi.org/10.1038/s41385-018-0085-5) contains supplementary material, which is available to authorized users.

Competing interests: The authors declare no competing interests.

\section{REFERENCES}

1. Siegel, R. L., Miller, K. D. \& Jemal, A. Cancer statistics, 2016. CA Cancer J. Clin. 66, 7-30 (2016).

2. Douaiher, J. et al. Colorectal cancer-global burden, trends, and geographical variations. J. Surg. Oncol. 115, 619-630 (2017).

3. Center, M. M., Jemal, A., Smith, R. A. \& Ward, E. Worldwide variations in colorectal cancer. CA Cancer J. Clin. 59, 366-378 (2009).

4. Siegel, R. L. et al. Colorectal cancer incidence patterns in the United States, 19742013. J Natl Cancer Inst. 109, 1-6 (2017).

5. Siegel, R. L., Miller, K. D. \& Jemal, A. Colorectal cancer mortality rates in adults aged 20 to 54 years in the United States, 1970-2014. JAMA 318, 572-574 (2017).

6. Mattar, M. C., Lough, D., Pishvaian, M. J. \& Charabaty, A. Current management of inflammatory bowel disease and colorectal cancer. Gastrointest. Cancer Res. 4, 53-61 (2011).

7. Reid, G. Probiotics: definition, scope and mechanisms of action. Best. Pract. Res. Clin. Gastroenterol. 30, 17-25 (2016).

8. Orel, R. \& Kamhi Trop, T. Intestinal microbiota, probiotics and prebiotics in inflammatory bowel disease. World J. Gastroenterol. 20, 11505-11524 (2014).

9. Ishikawa, $\mathrm{H}$. et al. Randomized trial of dietary fiber and Lactobacillus casei administration for prevention of colorectal tumors. Int. J. Cancer 116, 762-767 (2005).

10. Round, J. L. \& Mazmanian, S. K. Inducible Foxp3+ regulatory T-cell development by a commensal bacterium of the intestinal microbiota. Proc. Natl. Acad. Sci. USA 107, 12204-12209 (2010).

11. Round, J. L. et al. The Toll-like receptor 2 pathway establishes colonization by a commensal of the human microbiota. Science 332, 974-977 (2011).

12. Rabizadeh, S. et al. Enterotoxigenic bacteroides fragilis: a potential instigator of colitis. Inflamm. Bowel Dis. 13, 1475-1483 (2007).

13. Rhee, K. J. et al. Induction of persistent colitis by a human commensal, enterotoxigenic Bacteroides fragilis, in wild-type C57BL/6 mice. Infect. Immun. 77, 1708-1718 (2009)

14. $\mathrm{Wu}, \mathrm{S}$. et al. A human colonic commensal promotes colon tumorigenesis via activation of T helper type 17 T cell responses. Nat. Med. 15, 1016-1022 (2009).

15. Wu, S., Lim, K. C., Huang, J., Saidi, R. F. \& Sears, C. L. Bacteroides fragilis enterotoxin cleaves the zonula adherens protein, E-cadherin. Proc. Natl. Acad. Sci. USA 95, 14979-14984 (1998).
16. Wu, S., Morin, P. J., Maouyo, D. \& Sears, C. L. Bacteroides fragilis enterotoxin induces c-Myc expression and cellular proliferation. Gastroenterology 124, 392-400 (2003).

17. Chung, L. et al. Bacteroides fragilis toxin coordinates a pro-carcinogenic inflammatory cascade via targeting of colonic epithelial cells. Cell Host Microbe 23, 421 (2018).

18. Boleij, A. et al. The Bacteroides fragilis toxin gene is prevalent in the colon mucosa of colorectal cancer patients. Clin. Infect. Dis. 60, 208-215 (2015).

19. Dejea, C. M. et al. Patients with familial adenomatous polyposis harbor colonic biofilms containing tumorigenic bacteria. Science 359, 592-597 (2018).

20. Wick, E. C. et al. Stat3 activation in murine colitis induced by enterotoxigenic Bacteroides fragilis. Inflamm. Bowel Dis. 20, 821-834 (2014).

21. Mazmanian, S. K., Round, J. L. \& Kasper, D. L. A microbial symbiosis factor prevents intestinal inflammatory disease. Nature 453, 620-625 (2008).

22. Mazmanian, S. K., Liu, C. H., Tzianabos, A. O. \& Kasper, D. L. An immunomodulatory molecule of symbiotic bacteria directs maturation of the host immune system. Cell 122, 107-118 (2005).

23. Chae, W. J. et al. Ablation of IL-17A abrogates progression of spontaneous intestinal tumorigenesis. Proc. Natl. Acad. Sci. USA 107, 5540-5544 (2010).

24. Lee, S. M. et al. Bacterial colonization factors control specificity and stability of the gut microbiota. Nature 501, 426-429 (2013).

25. Huang, J. Y., Lee, S. M. \& Mazmanian, S. K. The human commensal Bacteroides fragilis binds intestinal mucin. Anaerobe 17, 137-141 (2011).

26. Roberton, A. M. \& Stanley, R. A. In vitro utilization of mucin by Bacteroides fragilis. Appl. Environ. Microbiol. 43, 325-330 (1982).

27. Tsai, H. H., Sunderland, D., Gibson, G. R., Hart, C. A. \& Rhodes, J. M. A novel mucin sulphatase from human faeces: its identification, purification and characterization. Clin. Sci. (Lond.) 82, 447-454 (1992).

28. Ki-Jong, R. et al. Induction of persistent colitis by a human commensal, enterotoxigenic Bacteroides fragilis, in wild-type C57BL/6 mice. Infect. Immun. 77, 1708-1718 (2009).

29. Hibbing, M. E., Fuqua, C., Parsek, M. R. \& Peterson, S. B. Bacterial competition: surviving and thriving in the microbial jungle. Nat. Rev. Microbiol. 8, 15-25 (2010).

30. Gillor, O., Etzion, A. \& Riley, M. A. The dual role of bacteriocins as anti- and probiotics. Appl. Microbiol. Biotechnol. 81, 591-606 (2008).

31. Chatzidaki-Livanis, M., Coyne, M. J. \& Comstock, L. E. An antimicrobial protein of the gut symbiont Bacteroides fragilis with a MACPF domain of host immune proteins. Mol. Microbiol. 94, 1361-1374 (2014).

32. Roelofs, K. G., Coyne, M. J., Gentyala, R. R., Chatzidaki-Livanis, M. \& Comstock, L. E. Bacteroidales secreted antimicrobial proteins target surface molecules necessary for gut colonization and mediate competition in vivo. MBio 7, pii: e01055-16 (2016).

33. Ochoa-Reparaz, J. et al. A polysaccharide from the human commensal Bacteroides fragilis protects against CNS demyelinating disease. Mucosal Immunol. 3, 487-495 (2010).

34. Macpherson, A. J. \& Harris, N. L. Interactions between commensal intestinal bacteria and the immune system. Nat. Rev. Immunol. 4, 478-485 (2004).

35. Chiu, C. C. et al. Monocolonization of germ-free mice with Bacteroides fragilis protects against dextran sulfate sodium-induced acute colitis. Biomed. Res. Int. 2014, 675786 (2014).

36. Chatzidaki-Livanis, M., Geva-Zatorsky, N. \& Comstock, L. E. Bacteroides fragilis type VI secretion systems use novel effector and immunity proteins to antagonize human gut Bacteroidales species. Proc. Natl. Acad. Sci. USA 113, 3627-3632 (2016).

37. Wexler, A. G. et al. Human symbionts inject and neutralize antibacterial toxins to persist in the gut. Proc. Natl. Acad. Sci. USA 113, 3639-3644 (2016).

38. Hecht, A. L. et al. Strain competition restricts colonization of an enteric pathogen and prevents colitis. EMBO Rep. 17, 1281-1291 (2016).

39. Zitomersky, N. L., Coyne, M. J. \& Comstock, L. E. Longitudinal analysis of the prevalence, maintenance, and IgA response to species of the order Bacteroidales in the human gut. Infect. Immun. 79, 2012-2020 (2011).

40. Basset, C., Holton, J., Bazeos, A., Vaira, D. \& Bloom, S. Are Helicobacter species and enterotoxigenic Bacteroides fragilis involved in inflammatory bowel disease? Dig. Dis. Sci. 49, 1425-1432 (2004).

41. Sears, C. L. Enterotoxigenic Bacteroides fragilis: a rogue among symbiotes. Clin. Microbiol. Rev. 22, 349-369 (2009).

42. Li, S. S. et al. Durable coexistence of donor and recipient strains after fecal microbiota transplantation. Science 352, 586-589 (2016).

43. Geis, A. L. et al. Regulatory T-cell response to enterotoxigenic Bacteroides fragilis colonization triggers IL17-dependent colon carcinogenesis. Cancer Discov. 5, 1098-1109 (2015).

44. Zitomersky, N. L. et al. Characterization of adherent bacteroidales from intestinal biopsies of children and young adults with inflammatory bowel disease. PLoS ONE 8, e63686 (2013). 
45. Papastathopoulou, A., Bezirtzoglou, E. \& Legakis, N. J. Bacterioides fragilis: production and sensitivity to bacteriocins. Anaerobe 3, 203-206 (1997).

46. Riley, T. V. \& Mee, B. J. A bacteriocin typing scheme for Bacteroides. J. Med. Microbiol. 15, 387-391 (1982).

47. Hillman, J. D., Mo, J., McDonell, E., Cvitkovitch, D. \& Hillman, C. H. Modification of an effector strain for replacement therapy of dental caries to enable clinical safety trials. J. Appl. Microbiol. 102, 1209-1219 (2007).

48. Gerding, D. N. et al. Administration of spores of nontoxigenic Clostridium difficile strain $\mathrm{M} 3$ for prevention of recurrent $\mathrm{C}$. difficile infection: a randomized clinical trial. JAMA 313, 1719-1727 (2015).
49. Coyne, M. J. et al. Polysaccharide biosynthesis locus required for virulence of Bacteroides fragilis. Infect. Immun. 69, 4342-4350 (2001).

50. Franco, A. A. The Bacteroides fragilis pathogenicity island is contained in a putative novel conjugative transposon. J. Bacteriol. 186, 6077-6092 (2004).

51. Tong, J., Liu, C., Summanen, P., Xu, H. \& Finegold, S. M. Application of quantitative real-time PCR for rapid identification of Bacteroides fragilis group and related organisms in human wound samples. Anaerobe 17, 64-68 (2011).

52. Dejea, C. M. et al. Microbiota organization is a distinct feature of proximal colorectal cancers. Proc. Natl. Acad. Sci. USA 111, 18321-18326 (2014). 